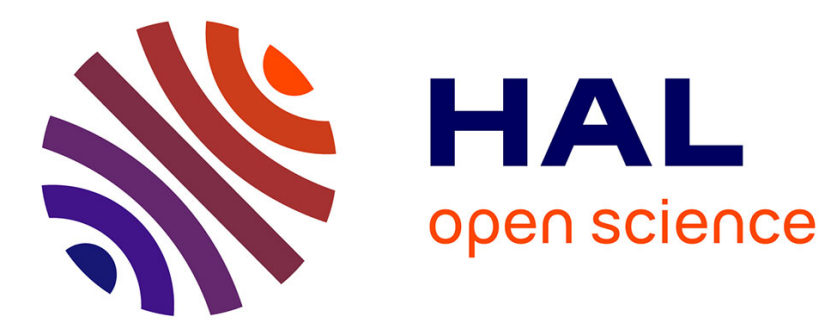

\title{
A consistent mass and momentum flux computation method for two phase flows. Application to atomization process
}

G. Vaudor, T. Ménard, W. Aniszewski, M. Doring, A. Berlemont

\section{- To cite this version:}

G. Vaudor, T. Ménard, W. Aniszewski, M. Doring, A. Berlemont. A consistent mass and momentum flux computation method for two phase flows. Application to atomization process. Computers and Fluids, 2017, 152, pp.204-216. 10.1016/j.compfluid.2017.04.023 . hal-01525891

\section{HAL Id: hal-01525891 https: / hal.sorbonne-universite.fr/hal-01525891}

Submitted on 22 May 2017

HAL is a multi-disciplinary open access archive for the deposit and dissemination of scientific research documents, whether they are published or not. The documents may come from teaching and research institutions in France or abroad, or from public or private research centers.
L'archive ouverte pluridisciplinaire HAL, est destinée au dépôt et à la diffusion de documents scientifiques de niveau recherche, publiés ou non, émanant des établissements d'enseignement et de recherche français ou étrangers, des laboratoires publics ou privés. 


\title{
A consistent mass and momentum flux computation method for two phase flows. Application to atomization process.
}

\author{
G. Vaudor ${ }^{\mathrm{a}}$, T. Ménard ${ }^{\mathrm{a}}$, W. Aniszewskic ${ }^{\mathrm{c}}$, M. Doring ${ }^{\mathrm{b}}$, A. Berlemont ${ }^{\mathrm{a}, *}$ \\ ${ }^{a}$ CNRS UMR 6614 - CORIA Normandie Université, CNRS, Université et INSA de Rouen, Site Universitaire du Madrillet, \\ Saint Etienne du Rouvray, France \\ ${ }^{b}$ LEMMA, 11 rue de Carnot 94270 Le Kremlin-Bicêtre, Paris, France \\ ${ }^{c}$ Sorbonne Universités, UPMC Univ Paris 06, CNRS UMR 7190, Institut Jean Le Rond d'Alembert, Paris, France
}

\begin{abstract}
This paper presents a new computational method for consistent calculation of mass and momentum fluxes in a two-phase flow simulation. The problem of inconsistency of the mass and momentum transfers has been long known in the two-phase flow context. Once the density ratio between fluid phases becomes high, and/or the momentum of one phase differs significantly from that of the other, a decoupling phenomenon causes a non-physical transfer of momentum, rendering most codes unstable. Original works of Rudman [31] have addressed this by proposing a way to couple the mass and momentum flux transport. To ensure this consistency between both fluxes in a staggered configuration, Rudman introduces a finer sub-grid to transport the volum of fluid.

In our paper, we present a way to adopt Rudman's approach without this subgrid, but always in a stagerred grid. The method is presented along with validation test cases and example applications, including very demanding momentum-dominated $3 \mathrm{D}$ simulations.
\end{abstract}

Keywords: Rudman technique, Two-phase flow, CLSVOF, Large Density Ratio, Coaxial liquid jet

\section{Introduction}

Many atomization processes are characterized by large density ratios coupled with large shear. A large velocity difference between fluid phases is required to destabilize the spray. For example, the fuel is injected at high velocity in Diesel engines but, in case of assisted atomization, found in cryotechnic applications, the liquid jet (ergol) is surrounded by a gas flow with quite high velocity to produce shear atomization. Numerical simulations of these configurations are known for becoming unstable. The main reason being insufficient coupling between mass and momentum numerical schemes.

In the VOF context, Rudman [31], in 1998, proposed an approach to ensure consistency between mass transport and momentum transport. This method allowed to perform two phase numerical simulations with high density ratio. The momentum flux calculations are directly obtained from VOF fluxes and require a finer sub-grid for the transport of the interface when using staggered grid. Many authors adapted this method to their numerical schemes. Among them, Bussman [22] adapted it to a collocated mesh and did not need subgrid strategy to transport the interface. This method is used by François \& al.[9] and with an unsplit VOF algorithm by Le Chenadec and al. [18]. More recently, the method was modified for Level Set (LS) algorithms $[28,7,12]$, in order to obtain the best approximation of flux momentum from the distance function. A simple LS approach was introduced by Raessi in 2009 [26, 7] but the flux calculations were one-dimensional and so did not take into account the orientation of the interface passing through the control

\footnotetext{
${ }^{*}$ Corresponding Author. email: berlemont@coria.fr; tel. +33 (0)2 329536 17, CNRS-UMR6614 CORIA, Universite de Rouen, Site Universitaire du Madrillet - BP 12, 76801 Saint Etienne du Rouvray cedex
} 
volume. Later, in 2010, a more sophisticated approach [27, 28] for two-dimensional cases was developed. Ghods and Herrmann [12] and Desjardins et al [6] succeeded in 2013 in proposing a three-dimensional discretization for flux calculation. Some of these methods were successfully applied to numerical simulation of liquid jet atomisation with high density ratio [6, 18, 28].

Sussman [33] introduced an extrapolated liquid velocity to provide stability in high speed flow with high density ratio, without previous treatment for the consistency between mass and momentum. The velocity field of the fluid with the largest density is extrapolated on some nodes in the lightest fluid, and introduced in the discretization of convective term. This method is also used by Li et al. [19] and Xiao et al. [36] to simulate the primary breakup of a liquid jet. The method is stable and gives good results. Desjardins and Moureau's approach [7] decouples gas and liquid velocities by introducing two distinct vector fields that are defined in all the domain. Velocity field of each phase is extended in the other phase thanks to the Aslam [2] method. Both fields are then advected and they are finally coupled through the pressure Poisson equation to obtain a single field.

Because our solver is a coupled levelset/VOF [25,3] on a staggered grid, the Rudman approach seems to be the best approch to ensure consistency between mass and momentum transport [35]. Nevertheless, the finer grid increases the computational cost of our CLSVOF method motivating the development of a single grid approach.

The structure of this paper is as follows: in the first section, we briefly present the numerical methods employed in our solver. Then the implementation of Rudman's method without a subgrid for the advection of the interface on a staggered formulation is detailled. Finally some test cases are presented validating the new method and showing the improvements when compare to previous results.

\section{Two phase incompressible Navier-Stokes Solver}

Interface advection.

The interface description is performed with a CLSVOF algorithm in which the Level Set function $\Phi$ describes geometric properties and the VOF function $C$ ensures mass conservation. Both functions are simultaneously advected as presented in $[34,25]$. Because the advection of VOF is the key point for the conservative mass/momentum advection method, it is briefly recalled how it is performed.

The equation to solve is :

which is split in (2D description):

for the $x$ direction :

$$
\frac{\partial C}{\partial t}+\nabla \cdot(C \mathbf{U})=C \nabla \cdot \mathbf{U}
$$

and for $y$ direction :

$$
\tilde{C}_{i, j}=\frac{C_{i, j}^{n}+\frac{\Delta t}{\Delta x}\left(f_{i-1 / 2, j}^{n}-f_{i+1 / 2, j}^{n}\right)}{1-\frac{\Delta t}{\Delta x}\left(u_{i+1 / 2, j}^{n}-u_{i-1 / 2, j}^{n}\right)}
$$

$$
\hat{C}_{i, j}=\frac{\tilde{C}_{i, j}+\frac{\Delta t}{\Delta y}\left(\tilde{f}_{i, j-1 / 2}-\tilde{f}_{i, j+1 / 2}\right)}{1-\frac{\Delta t}{\Delta y}\left(v_{i, j+1 / 2}^{n}-v_{i, j-1 / 2}^{n}\right)}
$$

At each solver time step, $\mathrm{x}-\mathrm{y}$ directions are switched.

The final expression of VOF is given by :

$$
C_{i, j}^{n+1}=\hat{C}_{i, j}-\hat{C}_{i, j} \frac{\Delta t}{\Delta y}\left(v_{i, j+1 / 2}^{n}-v_{i, j-1 / 2}^{n}\right)-\tilde{C}_{i, j} \frac{\Delta t}{\Delta x}\left(u_{i+1 / 2, j}^{n}-u_{i-1 / 2, j}^{n}\right)
$$

In Eq. (2), $f_{i+1 / 2, j}$ denotes the VOF flux through the face $(i+1 / 2, j)$ between the cells $(i, j)$ and $(i+1, j)$ :

$$
f_{i+1 / 2, j}=u_{i+1 / 2, j} c_{i+1 / 2, j}
$$


and $c_{i+1 / 2, j}$ represents a volume fraction (Fig. 1) defined by :

$$
c_{i+1 / 2, j}= \begin{cases}\frac{\int_{y_{j-1 / 2}}^{y_{j+1 / 2}} \int_{x_{i+1 / 2}-u_{i+1 / 2, j} \Delta t}^{x_{i+1 / 2}} H\left(\vec{n}_{i, j} \cdot\left(\vec{r}-\vec{r}_{i, j}\right)+\alpha_{i, j}\right) d x d y}{u_{i+1 / 2, j} \Delta t \Delta y} & \Leftrightarrow u_{i+1 / 2, j}>0 \\ \frac{\int_{y_{j-1 / 2}}^{y_{j+1 / 2}} \int_{x_{i+1 / 2}}^{x_{i+1 / 2}+\left|u_{i+1 / 2, j}\right| \Delta t} H\left(\vec{n}_{i+1, j} \cdot\left(\vec{r}-\vec{r}_{i+1, j}\right)+\alpha_{i+1, j}\right) d x d y}{\left|u_{i+1 / 2, j}\right| \Delta t \Delta y} & \Leftrightarrow u_{i+1 / 2, j}<0\end{cases}
$$

$H($.$) is the Heaviside function, the interface is represented with a linear segment in 2D (plane in 3D)$ whose equation is derived from on a linear Level Set recontruction $\Phi_{i, j}^{R}(\vec{r})=\vec{n}_{i, j} \cdot\left(\vec{r}-\vec{r}_{i, j}\right)+\alpha_{i, j}$. Here $\alpha_{i, j}$ represents the value of the reconstructed Level Set on the center point of the cell $\left(\vec{r}_{i, j}=\left(x_{i}, y_{j}\right)\right)$. The integrals in both equations can be computed with geometrical computations, e.g. using the VOF tools of Lopez \& Hernandez [21] or cube chopping method [13].

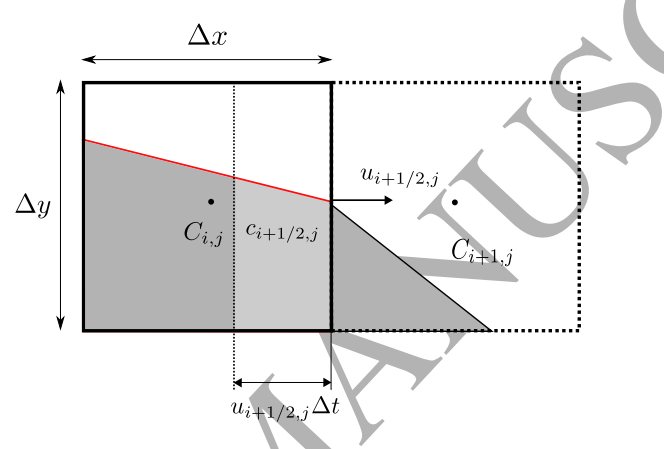

Figure 1: Schematic representation of $\widehat{V O F}$ advection on $x$-direction in the case of $u_{i+1 / 2}>0$

The density $\rho$ in Navier-Stokes equations is computed using the volume fraction [13] :

where

$$
\rho_{i+1 / 2, j}=C_{i+1 / 2, j} \rho_{l}+\left(1-C_{i+1 / 2, j}\right) \rho_{g}
$$

$$
\begin{aligned}
C_{i+1 / 2, j} & =C_{i+1 / 4, j}+C_{i+3 / 4, j} \\
& =\frac{1}{\Delta x \Delta y}\left(\int_{y_{j-1 / 2}}^{y_{j+1 / 2}} \int_{x_{i}}^{x_{i+1 / 2}} H\left(\Phi_{i, j}^{R}\right) d x d y+\int_{y_{j-1 / 2}}^{y_{j+1 / 2}} \int_{x_{i+1 / 2}}^{x_{i+1}} H\left(\Phi_{i+1, j}^{R}\right) d x d y\right)
\end{aligned}
$$

Note that, in our notation, $C_{i+1 / 4, j}$ and $C_{i+3 / 4, j}$ are between $[0,0.5]$.

Navier-Stokes equations resolution.

To obtain the pressure and velocity fields, the incompressible Navier-Stokes equations are solved :

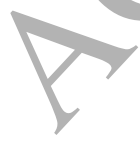

$$
\left\{\begin{array}{l}
\nabla \cdot \mathbf{U}=0 \\
\frac{\partial \mathbf{U}}{\partial t}=-(\mathbf{U} \cdot \nabla) \mathbf{U}+\frac{1}{\rho}(-\nabla P+\nabla \cdot(2 \mu \mathbf{D})+\mathbf{F})
\end{array}\right.
$$

Where

- $\mathbf{U}$ is the velocity field $(u, v, w)$.

- $\mu$ is the dynamic viscosity. 
- $\rho$ is the density.

- $P$ is the pressure.

- $\mathbf{D}$ is the strain rate tensor $\mathbf{D}=\frac{1}{2}\left(\nabla(\mathbf{U})+\nabla\left(\mathbf{U}^{T}\right)\right)$

- $\mathbf{F}=\mathbf{F}_{V}+\mathbf{F}_{S T}$ represents the body force and the surface tension force, $\mathbf{F}_{S T}=\sigma \kappa \delta_{I} \vec{n}$

$\sigma$ is the surface tension, $\kappa$ the curvature of the interface, $\vec{n}$ is the normal vector to the interface and $\delta_{I}$ is the Dirac function centered on it.

A standard projection method is used to solve the system. In a first step the momentum equation is solved without the pressure term to obtain an intermediate velocity $U^{*}$.

$$
\mathbf{U}^{*}=\mathbf{U}^{n}+\Delta t\left(-\left(\mathbf{U}^{n} \cdot \nabla\right) \mathbf{U}^{n}+\frac{1}{\rho^{n+1}} \nabla \cdot\left(2 \mu^{n} D\right)+\frac{1}{\rho^{n+1}} F\right)
$$

Which is linked to the pressure and velocity at time $n+1$ by :

$$
\mathbf{U}^{n+1}=\mathbf{U}^{*}+\frac{1}{\rho^{n+1}}(-\nabla P)
$$

We apply the divergence operator $(\nabla$.$) to the equation (10)$ to obtain the following implicit expression for pressure:

$$
\nabla \cdot\left(\frac{1}{\rho^{n+1}}\left(-\nabla P^{n+1}\right)\right)=\nabla \cdot\left(\mathbf{U}^{*}\right)
$$

Once the pressure field is determined, we are able to compute velocity field $\mathbf{U}^{n+1}$ from (10).

Viscosity and density are written as function of the phase indicator $(\phi)$ (VOF or Level Set).

The temporal integration is performed through a 2nd or 3rd order TVD Runge-Kutta scheme. The viscosity term is discretized following the method presented by Sussman [33]. The WENO scheme is implemented to discretize convective term in non-conservative form (U. $\nabla \mathbf{U})$ or in conservative form $\nabla$.(U $\otimes \mathbf{U})$. The Ghost-Fluid [8] method is employed in the spatial discretization of the Poisson equation (11) to take into account the surface tension force $F_{S T}$, treated as a jump of pressure. This equation is solved by a Conjugate Gradient preconditioned by a Multigrid Method (MGCG) [37].

\section{Development of the consistent, conservative mass/momentum advection method}

\subsection{Consistency between mass and momentum}

As mentioned by Rudman [31], the convective term should be written in the mass-conservative form to give consistency between mass and momentum transports. We focus on the mass transport equation and on the momentum equation (for clarity, we assume no viscosity, no gravity and no surface tension) :

$$
\left\{\begin{array}{c}
\frac{\partial \rho}{\partial t}+\nabla \cdot(\rho \mathbf{U})=0 \\
\frac{\partial \rho \mathbf{U}}{\partial t}+\nabla \cdot(\rho \mathbf{U} \otimes \mathbf{U})=(-\nabla P)
\end{array}\right.
$$

The mass transport equation (12a) is not explicitly solved, but it is deduced thanks to VOF advection equation $(1), \nabla \cdot \mathbf{U}=0$ property and the definition of density from $\operatorname{VOF}\left(\rho=C \rho_{l}+(1-C) \rho_{g}\right)$. 


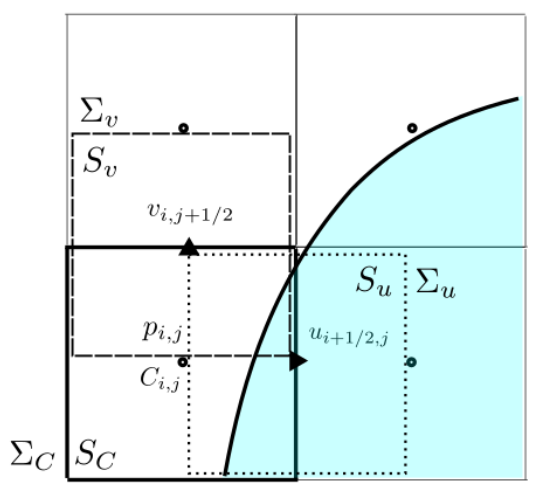

Figure 2: Representation of control volumes $(S)$ (and there corresponding contours $\Sigma$ ) for VOF/mass (black line) and for momentum ( $u$ : dotted line, $v$ : dashed line) on staggered grid

The convective term is discretized using a finite volume approach. For simplicity, description of the method is given for $2 \mathrm{D}$ configuration and thus integration of the system is given on a surface (control volume). We also apply the inverse Green-Ostrogradski theorem to the convective term to work with mass and momentum fluxes :

$$
\left\{\begin{array}{r}
\iint_{S_{C}} \frac{\partial \rho(C)}{\partial t} \mathrm{~d} S+\underbrace{\oint_{\Sigma_{C}}(\rho(C) \mathbf{U}) \cdot \vec{n} \mathrm{~d} \Sigma}_{I}=0 \\
\iint_{S_{u, v}} \frac{\partial \rho(C) \mathbf{U}}{\partial t} \mathrm{~d} S+\underbrace{\oint_{\Sigma_{u, v}}(\mathbf{U} \otimes \rho(C) \mathbf{U}) \cdot \vec{n} \mathrm{~d} \Sigma}_{I I}=\iint_{S_{u, v}}(-\nabla P) \mathrm{d} S
\end{array}\right.
$$

Equation (13b) leads to two expressions for $u$ and $v$ velocity components. In the staggered grid approach velocity components are defined at the center of control volume faces, thus equations (13a and 13b) are spatially integrated around different control volumes $\left(S_{u}, S_{v}\right)$ centered on velocity $u$ and $v($ Fig. 2$)$. The mass equation (13a) (deduced from VOF equation (1)) is integrated around a control volume $\left(S_{C}\right)$ centered on VOF value.

Discretization must be carried out with consistency between the transport of mass and momentum. We note that the mass flux $(I)$ and the momentum flux $(I I)$ in Eqs. (13a) and (13b) have common term $\rho(C) U$. The mass flux $(I)$ is computed using VOF advection and must be included in the momentum flux $(I I)$ to ensure consistency, but their respective control volumes are different in a staggered grid strategy. This is why Rudman introduces a refined grid for VOF (Fig. $3 \mathrm{rhs)} \mathrm{which} \mathrm{is} \mathrm{not} \mathrm{required} \mathrm{when} \mathrm{a} \mathrm{collocated} \mathrm{grid} \mathrm{is}$ used $[22,9]$ (Because $\Sigma_{C}=\Sigma_{u}=\Sigma_{v}$ ) (Fig. 3 lhs).

In the three following sections (3.2-3.4), we present our approach to avoid the subgrid (Fig. 3 center). We detail how to extract mass fluxes from VOF fluxes on the faces of $u$ and $v$ control volume to inject them in the momentum equations. 

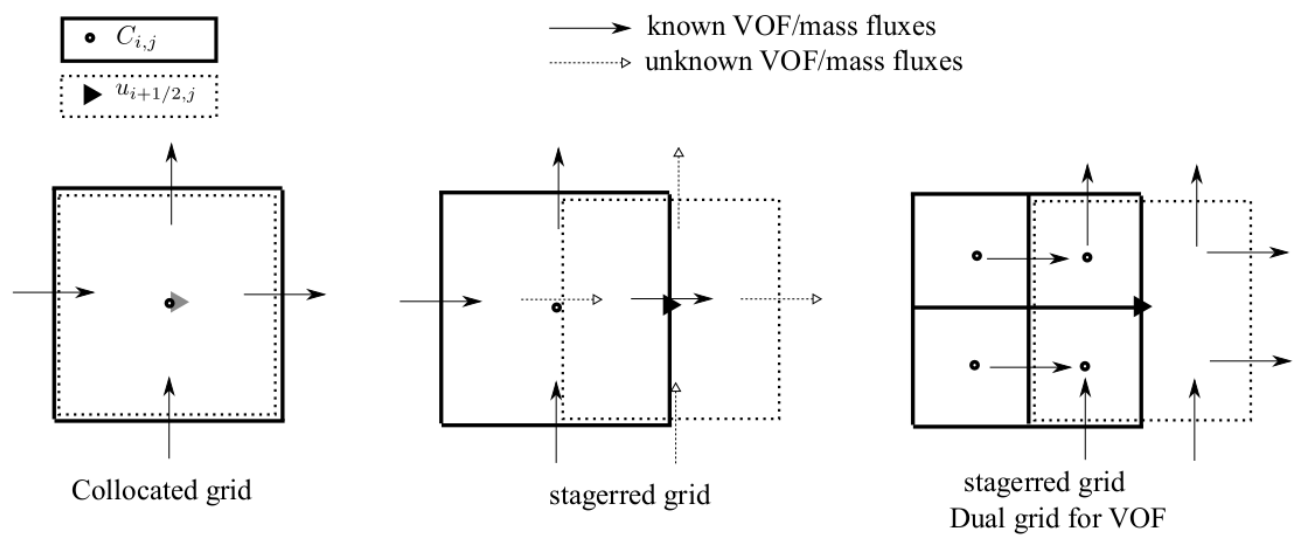

Figure 3: Representation of known and unknown VOF (mass) fluxes on cellfaces is the case of collocated grid, staggered grid, and staggered with a dual grid for VOF advection. Only $u$ and C control volumes are shown.

\subsection{Mass flux computation}

The equivalence of mass and VOF allows to deduce the mass flux (noted $f^{m, x}=\rho u$ for $\mathrm{x}$-direction and $f^{m, y}=\rho v$ for $\mathrm{y}$-direction) from VOF flux (previously noted $f=u \times c$ ) during the interface advection. For face $(i+1 / 2, j)$, located on the right side of $\Sigma_{C}$, we have the following relation :

$$
f_{i+1 / 2, j}^{m, x}=u_{i+1 / 2, j}\left(c_{i+1 / 2, j} \rho_{l}+\left(1-c_{i+1 / 2, j}\right) \rho_{g}\right)=f_{i+1 / 2, j} \rho_{l}+\left(u_{i+1 / 2, j}-f_{i+1 / 2, j}\right) \rho_{g}
$$

Our strategy to obtain mass flux through the outline of $S_{u}$ and $S_{v}$ with a single grid is now detailed. It is split in two steps. The first step describes how to compute mass fluxes on surface given by $\Sigma_{C} \cap \Sigma_{u}$ (and $\Sigma_{C} \cap \Sigma_{v}$ ) during advection of the interface. In the second we compute mass fluxes on the part of $\Sigma_{u}$ (and $\Sigma_{v}$ ) given by $\left.\Sigma_{u}\right|_{i+1 / 2, j}-\left.\left.\Sigma_{C}\right|_{i, j} \cup \Sigma_{C}\right|_{i+1, j}$, noted later $\Sigma_{u}-\Sigma_{C}$, (respectively $\Sigma_{v}-\Sigma_{C}$ ), performed when the advection of interface is finished.

- First, the term $c_{i+1 / 2, j}$ is written as (Fig. 4):

$$
c_{i+1 / 2, j}=c_{i+1 / 2, j-1 / 4}+c_{i+1 / 2, j+1 / 4}
$$

if $u_{i+1 / 2, j}>0$ is considered, the expression of the right side terms of Eq. 15 are :

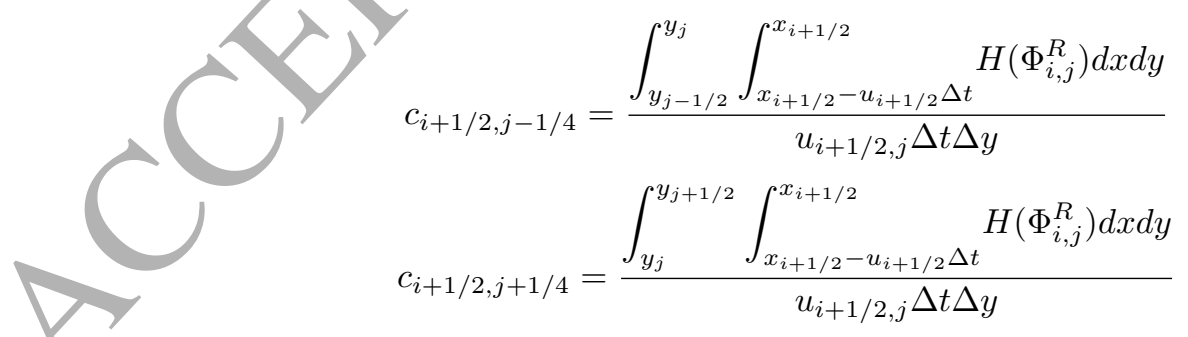




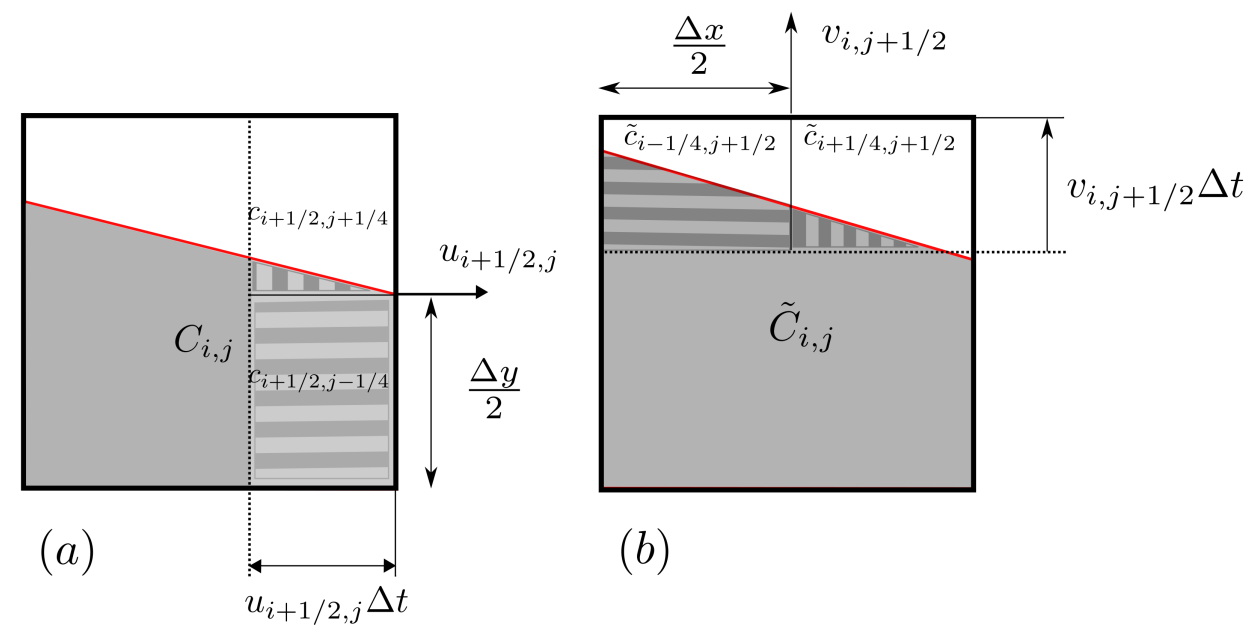

Figure 4: (a) Representation of $c_{i+1 / 2, j-1 / 4}$ and $c_{i+1 / 2, j+1 / 4}$ (respectively horizontal and vertical bands) normalized by $u_{i+1 / 2, j} \Delta t \Delta y$, (b) $\tilde{c}_{i-1 / 4, j+1 / 2}$ and $\tilde{c}_{i+1 / 4, j+1 / 2}$ normalized by $v_{i, j+1 / 2} \Delta t \Delta x$

The corresponding VOF flux is given by :

$$
f_{i+1 / 2, j}=f_{i+1 / 2, j-1 / 4}+f_{i+1 / 2, j+1 / 4}
$$

where $f_{i+1 / 2, j \pm 1 / 4}=u_{i+1 / 2, j} \times c_{i+1 / 2, j \pm 1 / 4}$ and the corresponding mass flux :

$$
f_{i+1 / 2, j \pm 1 / 4}^{m, x}=\rho_{l} f_{i+1 / 2, j \pm 1 / 4}+\rho_{g}\left(\frac{u_{i+1 / 2, j}}{2}-f_{i+1 / 2, j \pm 1 / 4}\right)
$$

The total mass flux on face $(i+1 / 2, j)$ is :

$$
f_{i+1 / 2, j}^{m, x}=f_{i+1 / 2, j-1 / 4}^{m, x}+f_{i+1 / 2, j+1 / 4}^{m, x}
$$

Once this procedure is performed on faces $(i+1 / 2, j)$ and $(i, j+1 / 2)$, the following mass fluxes are known on each cell : $f_{i+1 / 2, j-1 / 4}^{m, x}, f_{i+1 / 2, j+1 / 4}^{m, x}, f_{i+1 / 4, j+1 / 2}^{m, y}$ and $f_{i-1 / 4, j+1 / 2}^{m, y}$. as shown on Fig. 5 . Note that the left and bottom faces have similar fluxes incoming by neighbouring cells.

If we observe the control volume of $u$ (Fig. 6), only two faces have unknown fluxes (same in 3D) corresponding to the part of $\Sigma_{u}$ given by $\Sigma_{u}-\Sigma_{C}$. 


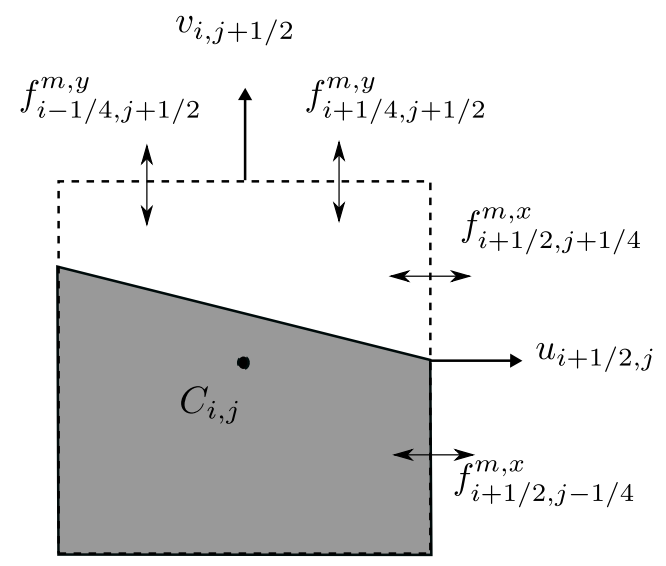

Figure 5: Representation of mass flux computed for each cell

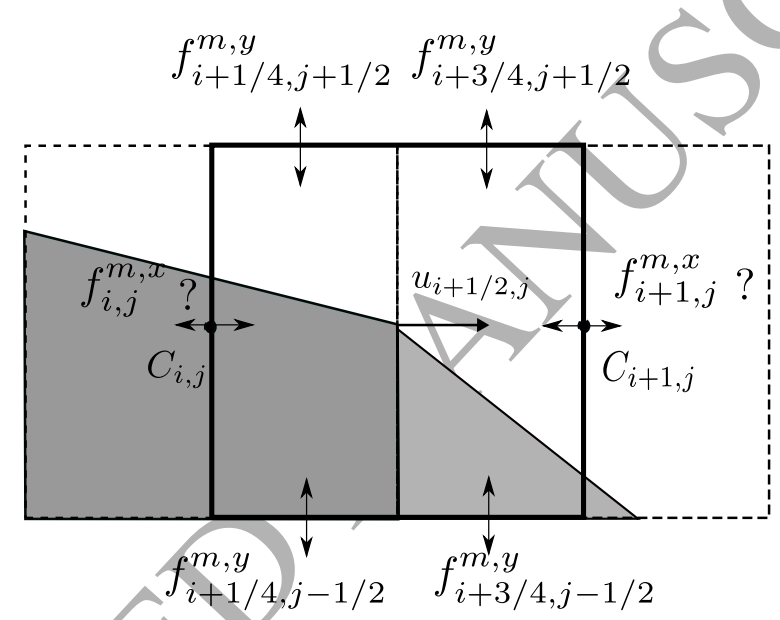

Figure 6: Representation of u control volume with known and unknown fluxes (denotes by question symbol)

- To calculate the first missing flux, a balance of mass is performed on a half-cell (Fig. 7). For the left part of control volume $S_{u}$, we obtain :

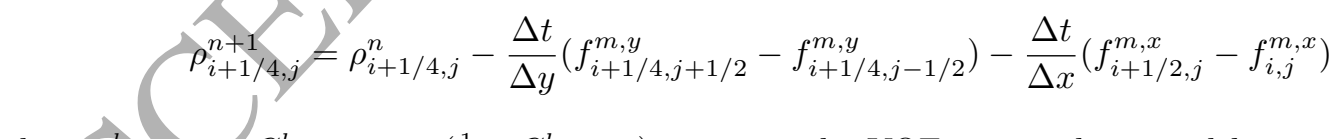

where $\rho_{i+1 / 4, j}^{l}=C_{i+1 / 4, j}^{l} \rho_{l}+\left(\frac{1}{2}-C_{i+1 / 4, j}^{l}\right) \rho_{g}$ is given by VOF at time $l=n$ and $l=n+1$ (note that $\rho_{i+1 / 4, j}^{l} \in\left[\rho_{g} / 2, \rho_{l} / 2\right]$, see remarks at the end of this section).

Because the advection of interface is performed, the only unknown term in Eq. (21) is $f_{i, j}^{m, x}$ which can thus be easily deduced.

The last unknown flux $f_{i+1, j}^{m, x}$ is computed in the same way when the balance of mass is performed on the left part of the control volume $S_{u}$ of the neighbouring cell $u_{i+3 / 2, j}$. 

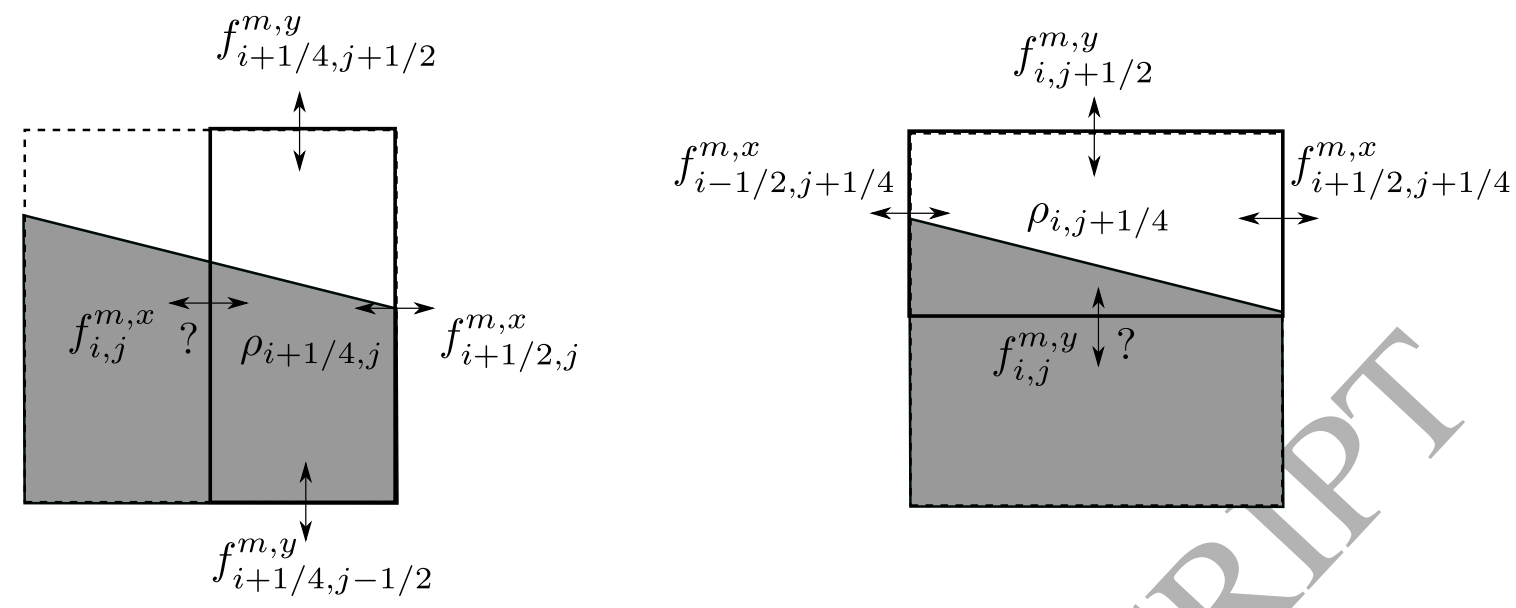

Figure 7: Representation of half control volume of $u$ and $v$ where mass balance is done. Known and unknown fluxes are shown.

Remarks:

1. The VOF transport is not changed, but $c_{i+1 / 2, j}$ is now split in two parts $\left(c_{i+1 / 2, j+1 / 4}\right.$ and $\left.c_{i+1 / 2, j-1 / 4}\right)$. To simplify implementation, with $c_{i+1 / 2, j}$ already computed for VOF advection, $c_{i+1 / 2, j+1 / 4}$ is computed as the same time. That means VOF fluxes $\left(f_{i+1 / 2, j}\right.$ and $\left.f_{i+1 / 2, j+1 / 4}\right)$ and mass fluxes $\left(f_{i+1 / 2, j}^{m, x}\right.$ and $\left.f_{i+1 / 2, j+1 / 4}^{m, x}\right)$ can be directly deduced at the same time.

To compute any flux on the face $(i+1 / 2, j-1 / 4)$ we use Eqs. (15,18 and 20) as they are required later for momentum flux computation (Eqs. (26) and (27)).

2. The above description concerns face $(i+1 / 2, j)$. It can be easily generalized for the face $(i, j+1 / 2)$. The other face $(i-1 / 2, j)$ and $(i, j-1 / 2)$ quantities are obtained by neighbouring cells. As it is done in regular VOF solvers.

3. It is important to note that we made the choice to define some variables as "half quantities" : thus $c_{. \pm 1 / 4} \in[0,0.5]$ (thus $f_{. \pm 1 / 4}$ and $\left.f_{. \pm 1 / 4}^{m, .}\right), C_{. \pm 1 / 4} \in[0,0.5]$ and $\rho_{. \pm 1 / 4} \in\left[\rho_{g} / 2, \rho_{l} / 2\right]$. It is linked to the computation of mass balance on a half cell. Indeed, if we write the equation of mass transport Eq. (13a) on a half-cell, we have :

$$
\frac{\left.\partial \rho\right|_{i+1 / 4, j}}{\partial t} \frac{\Delta x}{2} \Delta y+\left(\left.\rho v\right|_{i+1 / 4, j+1 / 2}-\left.\rho v\right|_{i+1 / 4, j-1 / 2}\right) \frac{\Delta x}{2}+\left(\left.\rho u\right|_{i+1 / 2, j}-\left.\rho u\right|_{i, j}\right) \Delta y=0
$$

The coefficients $\frac{1}{2}$ are included in the density to obtain :

$$
\text { with } \frac{\partial \rho_{i+1 / 4, j}}{\partial t} \Delta x \Delta y+\left(f_{i+1 / 4, j+1 / 2}^{m, y}-f_{i+1 / 4, j-1 / 2}^{m, y}\right) \Delta x+\left(f_{i+1 / 2, j}^{m, x}-f_{i, j}^{m, x}\right) \Delta y=0
$$

4. In 3D, both with $c_{i+1 / 2, j+1 / 4, k}$ and $c_{i+1 / 2, j-1 / 4, k}$, two more fluxes are necessary on the face $(i+$ $1 / 2, j, k)$, namely $c_{i+1 / 2, j, k+1 / 4}$ and $c_{i+1 / 2, j, k-1 / 4}$. That means four integrals are needed on each face (Fig. 8). 

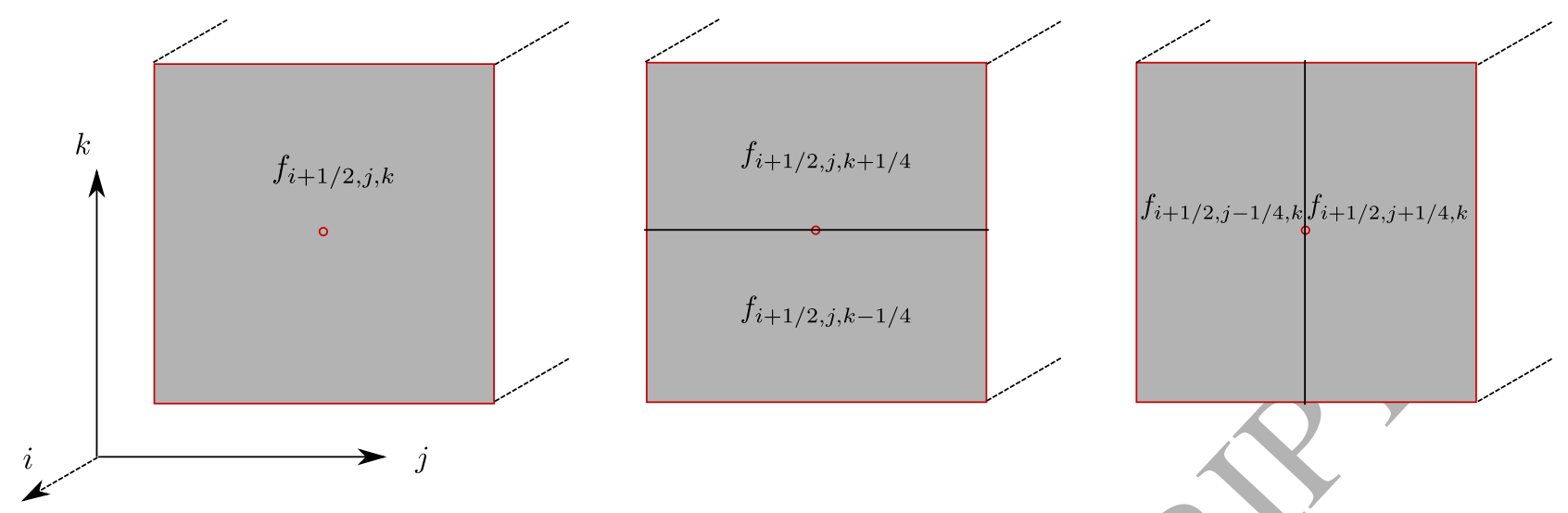

Figure 8: Representation of the face $(i+1 / 2, j, k)$ in $3 D$ with the areas of necessary fluxes.

Noting that:

$$
c_{i+1 / 2, j, k}=c_{i+1 / 2, j+1 / 4, k}+c_{i+1 / 2, j-1 / 4, k}=c_{i+1 / 2, j, k+1 / 4}+c_{i+1 / 2, j, k-1 / 4}
$$

we can compute only three above terms, $c_{i+1 / 2, j, k}, c_{i+1 / 2, j+1 / 4, k}$ and $c_{i+1 / 2, j, k+1 / 4}$. (Remark 1 holds) We recall that (for $u_{i+1 / 2, j, k}>0$ ) :

$$
\begin{gathered}
c_{i+1 / 2, j+1 / 4, k}=\frac{\int_{z_{k-1 / 2}}^{z_{k+1 / 2}} \int_{y_{j}}^{y_{j+1 / 2}} \int_{x_{i+1 / 2}-u_{i+1 / 2, j, k} \Delta t}^{x_{i+1 / 2}} H\left(\Phi_{i, j, k}^{R}\right) d x d y d z}{u_{i+1 / 2, j, k} \Delta t \Delta y \Delta z} \\
c_{i+1 / 2, j, k+1 / 4}=\frac{\int_{z_{k}}^{z_{k+1 / 2}} \int_{y_{j-1 / 2}}^{y_{j+1 / 2}} \int_{x_{i+1 / 2}-u_{i+1 / 2, j, k} \Delta t}^{x_{i+1 / 2}} H\left(\Phi_{i, j, k}^{R}\right) d x d y d z}{u_{i+1 / 2, j, k} \Delta t \Delta y \Delta z}
\end{gathered}
$$

\subsection{Momentum flux computation}

All mass fluxes are now known on all faces of $u$ and $v$ control volumes. They are now used for the computation of the convective terms II in Eq. (13b):

$$
\begin{aligned}
& \oint_{\Sigma_{u}}(u \otimes \rho \mathbf{U}) \cdot \vec{n} \mathrm{~d} \Sigma=\Delta y\left(\bar{u}_{i+1, j} f_{i+1, j}^{m, x}-\bar{u}_{i, j} f_{i, j}^{m, x}\right) \\
& +\Delta x\left(\bar{u}_{i+1 / 2, j+1 / 2}\left(f_{i+1 / 4, j+1 / 2}^{m, y}+f_{i+3 / 4, j+1 / 2}^{m, y}\right)-\bar{u}_{i+1 / 2, j-1 / 2}\left(f_{i+1 / 4, j-1 / 2}^{m, y}+f_{i+3 / 4, j-1 / 2}^{m, y}\right)\right) \\
& \oint_{\Sigma_{v}}(v \otimes \rho \mathbf{U}) \cdot \vec{n} \mathrm{~d} \Sigma=\Delta x\left(\bar{v}_{i, j+1} f_{i, j+1}^{m, y}-\bar{v}_{i, j} f_{i, j}^{m, y}\right) \\
& +\Delta y\left(\bar{v}_{i+1 / 2, j+1 / 2}\left(f_{i+1 / 2, j+1 / 4}^{m, x}+f_{i+1 / 2, j+3 / 4}^{m, x}\right)-\bar{v}_{i-1 / 2, j+1 / 2}\left(f_{i-1 / 2, j+1 / 4}^{m, y}+f_{i-1 / 2, j+3 / 4}^{m y}\right)\right)
\end{aligned}
$$


where $\bar{u}$ and $\bar{v}$ are unknown velocities which are interpolated, using a WENO 5 scheme [15] to ensure a higher interpolation order even close to the interface. However, our scheme is coupled with a limiter (first order upwind scheme) near interface (less than $2 \Delta x$ ).

In the projection method, the intermediate velocity $U^{*}$ is then obtained by :

$$
\mathbf{U}^{*}=\frac{1}{\rho^{n+1}}\left(\rho^{n} \mathbf{U}^{n}-\frac{\Delta t}{V} \oint_{\Sigma}(\mathbf{U} \otimes \rho \mathbf{U})^{n} \vec{n} \mathrm{~d} \Sigma\right)
$$

with $V=\Delta x \times \Delta y$.

\subsection{General algorithm}

To summarize, we rephrase the complete procedure for $2 \mathrm{D}$ case, representing a classical Euler step. All following points should successively be performed for all cells and/or faces :

1. Compute the densities $\left(\rho_{i+1 / 2, j}^{n}\right.$ and $\left.\rho_{i, j+1 / 2}^{n}\right)$ at time $n$ for control volumes $S_{u}, S_{v}$ and for a half part of these, for example the left part and bottom part : $\rho_{i+1 / 4, j}^{n}$ and $\rho_{i, j+1 / 4}^{n}$.

2. Advect the VOF with the "split" algorithm. Apart from classical VOF fluxes $f_{i+1 / 2, j}$ and $\tilde{f}_{i, j+1 / 2}$, compute the additional half-fluxes $f_{i+1 / 2, j+1 / 4}$ and $\tilde{f}_{i+1 / 4, j+1 / 2}$.

3. Compute corresponding mass fluxes $f_{i+1 / 2, j}^{m, x}, f_{i, j+1 / 2}^{m, y}$ using Eq. (14) and $f_{i+1 / 4, j+1 / 2}^{m, y}, f_{i+1 / 2, j+1 / 4}^{m, x}$ from Eq. (19) .

4. Repeat step 1 for time $n+1$.

5. Deduce fluxes $f_{i, j}^{m, x}$ and $f_{i, j}^{m, y}$ from balance of mass on half cells (Eq. (21)).

6. Deduce mass fluxes $f_{i-1 / 4, j+1 / 2}^{m, y}, f_{i+1 / 2, j-1 / 4}^{m, x}$ from (Eq. (20))

7. Compute interpolated velocity $\bar{u}$ on faces $(i, j)$ and $(i+1 / 2, j+1 / 2)$, and $\bar{v}$ on faces $(i, j)$ and $(i+$ $1 / 2, j+1 / 2)$.

8. Compute convective terms given by Eqs. (26) and (27)

9. Compute $U^{*}$ using Eq. (28)

10. Compute $p^{n+1}$ using Eq. (11)

11. Compute $U^{n+1}$ using Eq. (10)

This Euler step is then used in a predictor-corrector method instead of Runge-Kutta TVD schemes.

\section{Numerical results}

\subsection{High density droplet}

This test case was first introduced by Bussmann [22] in 2002, and is described in several papers [7, 28, 12] dealing with high density ratio flows in context of momentum conservation. Indeed, the test case is characterized by a very high density ratio $\left(1: 10^{6}\right)$ between the liquid droplet and air. Initially, a droplet of radius $R=0.1 \mathrm{~m}$ is located at the center of a square field with $L=1 \mathrm{~m}$. Velocity is initialized as:

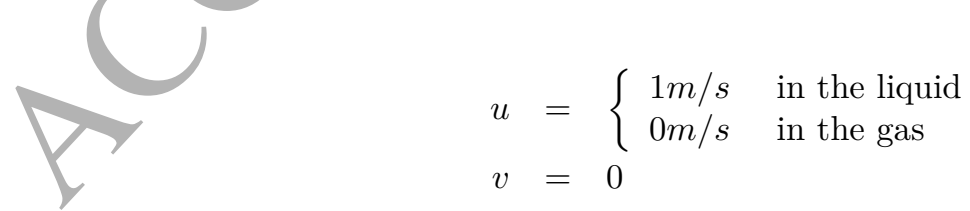

Boundary conditions are periodic. Surface tension force, viscous forces and body forces are all assumed zero. Ideally, after 1 second, the droplet must have completed a full turnover and thus recover its initial position with no deformation. This is however possible only if numerical scheme is adapted to significant density ratios.

Four discretizations of the convective term are compared here: the non-conservative and conservative forms (which were both originally implemented in the Archer3d solver), the original Rudman's method with 
a refine grid for VOF transport and the new method using a single grid. Simulations are performed on three different grids: $16 \times 16,32 \times 32,64 \times 64$. For the original Rudman's method, the mesh size is given for Navier-Stokes equation (no-refine grid), then it is twice finer for interface (VOF and Level Set). However, following representation of interface (represented by zero level set curve) and error computation are done on the Navier-Stokes mesh.

In Fig. 9 results are given for non-conservative and conservative forms of convective term, the droplet is strongly deformed and no grid convergence is observed, illustrating the failure of standard discretization for high density ratio configuration.
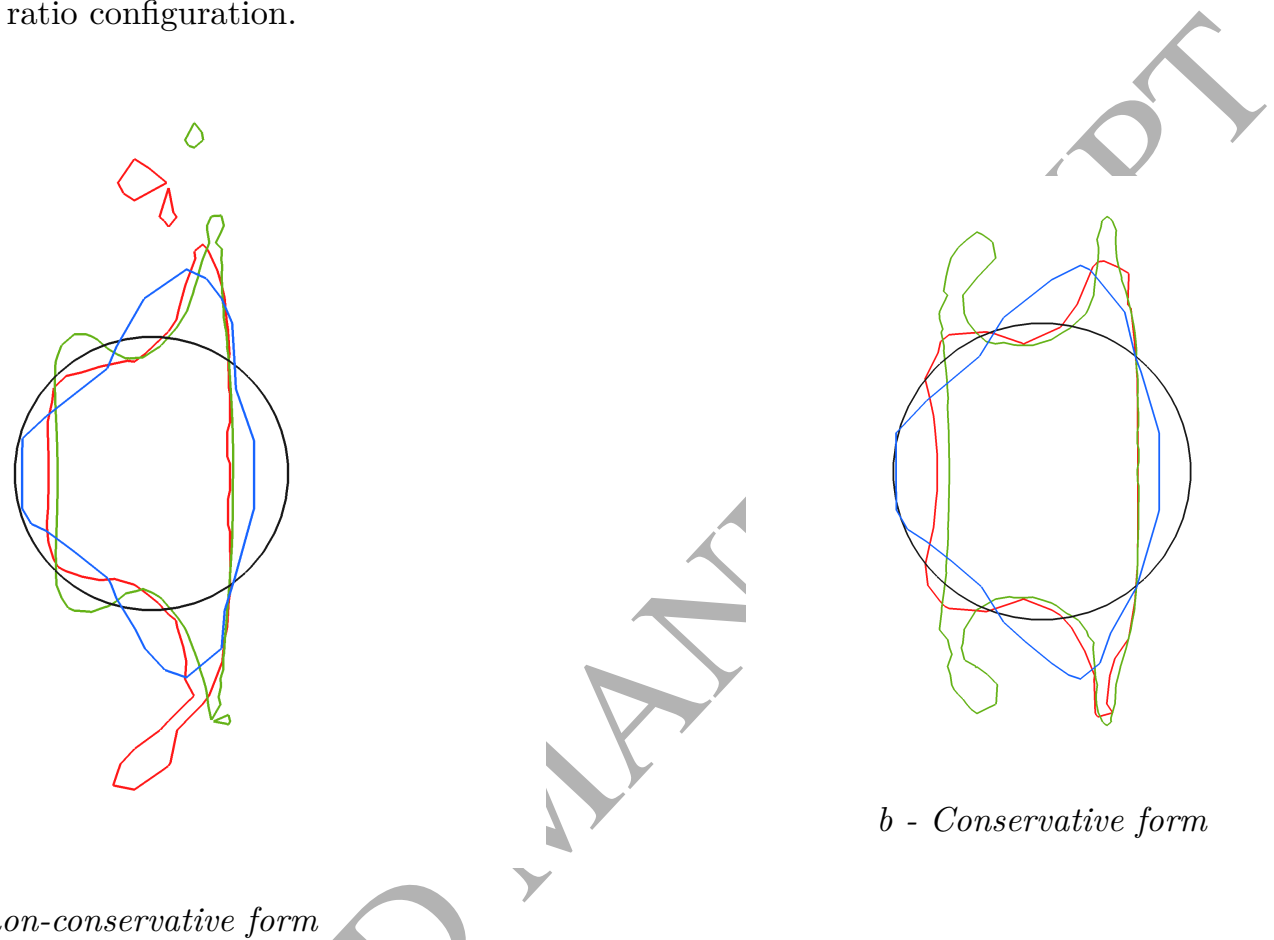

a- non-conservative form

Figure 9: In black the reference shape, blue is $16 x 16$, red is $32 x 32$ and green is $64 x 64$

Figure 10 presents the results with original Rudman's method and with the new scheme (grids up to $128^{2}$ are presented). In both cases, results are much better than those obtained with previous discretization. Moreover, increasing the resolution improves the quality of the solution as visible in the enlargements presented in Fig. 10.

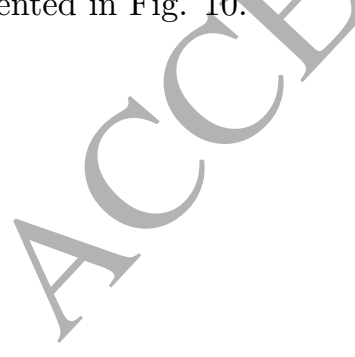




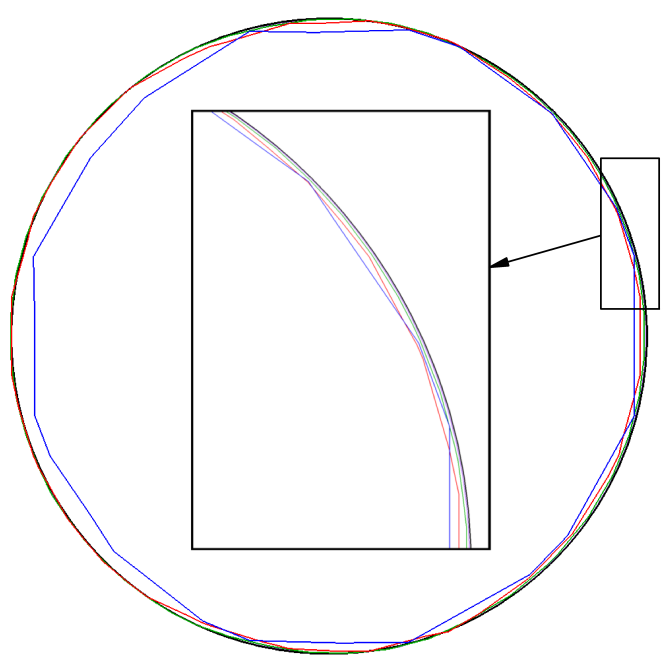

a - Interface after one lap with original Rudman method with subgrid for VOF.

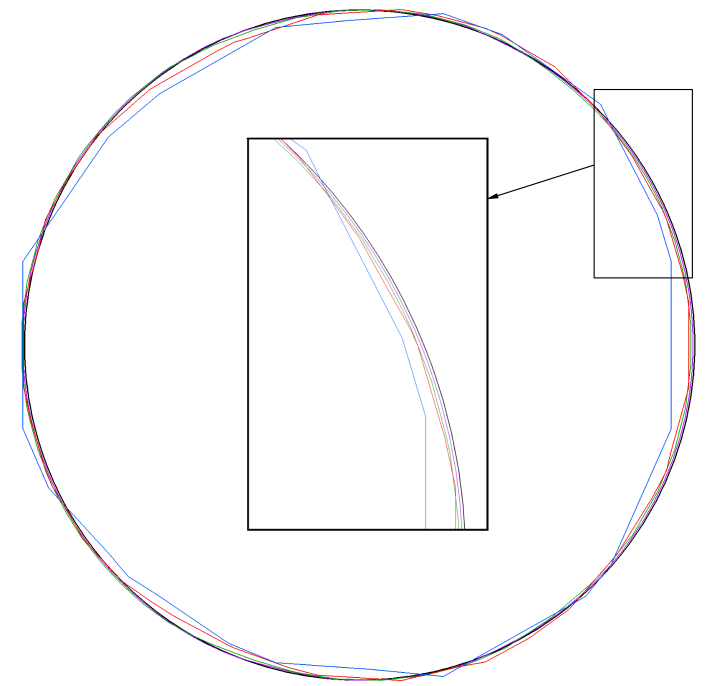

$b$ - Interface after one lap with the conservative momentum method on single grid

Figure 10: Initial and final stages of the droplet advection. The reference shape is drawn with black line, blue is the result on a $16^{2}$ grid, red $32^{2}$, green $64^{2}$ with the $128^{2}$ result shown in violet. The insets show a zoomed region.

To quantify this improvement, we present, in Table 1 advection errors measured as differences between original and final interface positions ${ }^{1}$. Subsequent table rows present varying grid refinements up to $128^{3}$. It is easily noticeable that both variants of Rudman method present convergence of (roughly) first order and comparable error values.

Table 1: The convergence of advection error for the high-density droplet test case. Approximate order convergence is given for both the single-grid method and the original (dual grid) Rudman's method.

\begin{tabular}{ccccc} 
Gridpoints & New Method & Order & Dual-Grid & Order \\
\hline $16^{2}$ & $2.7 \cdot 10^{-2}$ & - & $3.2 \cdot 10^{-2}$ & - \\
$32^{2}$ & $1.3 \cdot 10^{-2}$ & 1.03 & $1.15 \cdot 10^{-2}$ & 1.39 \\
$64^{2}$ & $5.0 \cdot 10^{-3}$ & 1.3 & $3.7 \cdot 10^{-3}$ & 1.55 \\
$128^{2}$ & $1.7 \cdot 10^{-3}$ & 1.47 & $1.2 \cdot 10^{-3}$ & 1.54
\end{tabular}

The high-density droplet advection test case has been also utilized to measure any differences in CPU cost between the newly proposed, single grid method implementation and the clasical dual grid variant. This has been performed by measuring the wall-clock time duration $t_{\text {iter }}$ of entire solver iteration over entire simulation, for both methods, using the $64^{2}$ grid. Mean values $\left\langle t_{i t e r}\right\rangle^{1}$ and $h\left\langle t_{i t e r}\right\rangle^{2}$ for single- and dual-grid methods respectively have been found, showing that

$$
\frac{<t_{i t e r}>^{1}}{<t_{\text {iter }}>^{2}} \approx 0.59
$$

\footnotetext{
${ }^{1}$ The $L_{1}$ norm of difference between VOF fraction function values for $t=0 \mathrm{~s}$ and $t=1 \mathrm{~s}$.
} 
meaning that a $41 \%$ gain can be attributed to application of the new method. This estimation is however coarse, the CLSVOF method is solved on the twice finer grid for original rudman's technics in all the domain, that means LS and VOF transport and redistance algorithm.

\subsection{Droplet oscillation}

In this second test case, we study the oscillations of a viscous, two-dimensional droplet due to surface tension effects. The same test case was previsouly presented by Raessi and Pitsch [28] to show the improvements in energy conservation with their momentum conserving method. Note that the same case has been extensively studied (using Archer with classical momentum schemes) by Aniszewski et al. in 3-D [1].

The droplet is initialized as an ellipse with semi-major $x$ and semi-minor $y$ axes of 0.15 and 0.1 respectively, located at the center of a square domain of side 1. Physical properties of the fluids are reported in Table 2 .

\begin{tabular}{|c|c|c|}
\hline & liquid & gas \\
\hline$\rho$ & 1000 & 1 \\
\hline$\mu$ & $7.510^{-3}$ & $7.510^{-3}$ \\
\hline$\sigma$ & \multicolumn{2}{|c|}{0.1} \\
\hline
\end{tabular}

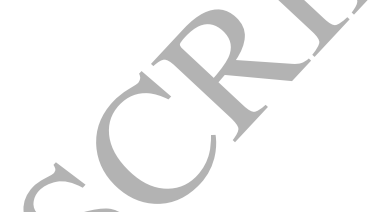

Table 2: Physical properties of liquid and gas

As viscous forces damp the energy of the system, the oscillation amplitude decreases with time. Applying the theoretical work of Rush \& Nadim [32] and Lamb [14], we present the temporal evolution of total energy:

$$
E(t)=E_{0} e^{-2 n(n-1) \nu^{*} t}
$$

where $n$ stands for the oscillation mode number, $t$ for time, and $\nu^{*}$ is a nondimensionalized viscosity scaled by the ratio of timescales specific to diffusion and oscillation: $\nu^{*}=\mu_{l} / \sqrt{\rho_{l} \sigma R}$. $R$ is the radius of the droplet when it is circular. In the results below, we use the dominant, second oscillation mode, so $n=2$.

The Figure 11 presents the comparison between the analytical total energy formula and the value of kinetic energy for our different convective methods along the time. Note that energy is non-dimensionalized by the initial energy $E_{0}$. The uniform mesh resolution is $64 \times 64$. Obviously the $E$ and $E_{k}$ are comparable only when the latter is maximum and $E_{\hat{p}}=0$, i.e. at $E_{k}$ peaks. We observe a better fit to the analytical curve once we adopt the conservative momentum scheme for the convective term. The non-conservative form, which produces too high energy dissipation, performs the worst. 


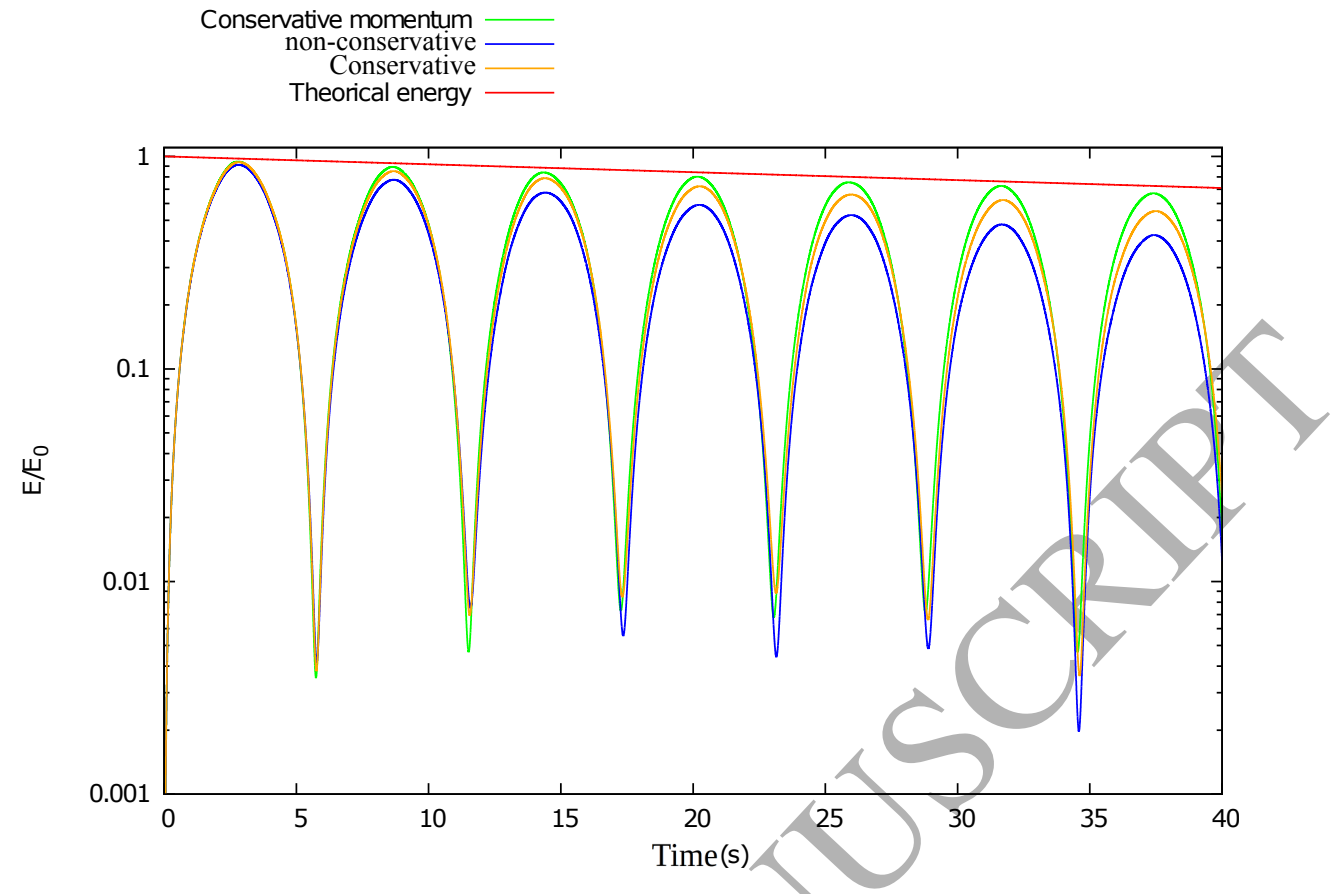

Figure 11: non-dimensional theorical energy and non-dimensional kinetic energic for: conservative momentum method(green), conservative form (yellow), non-conservative form (blue).

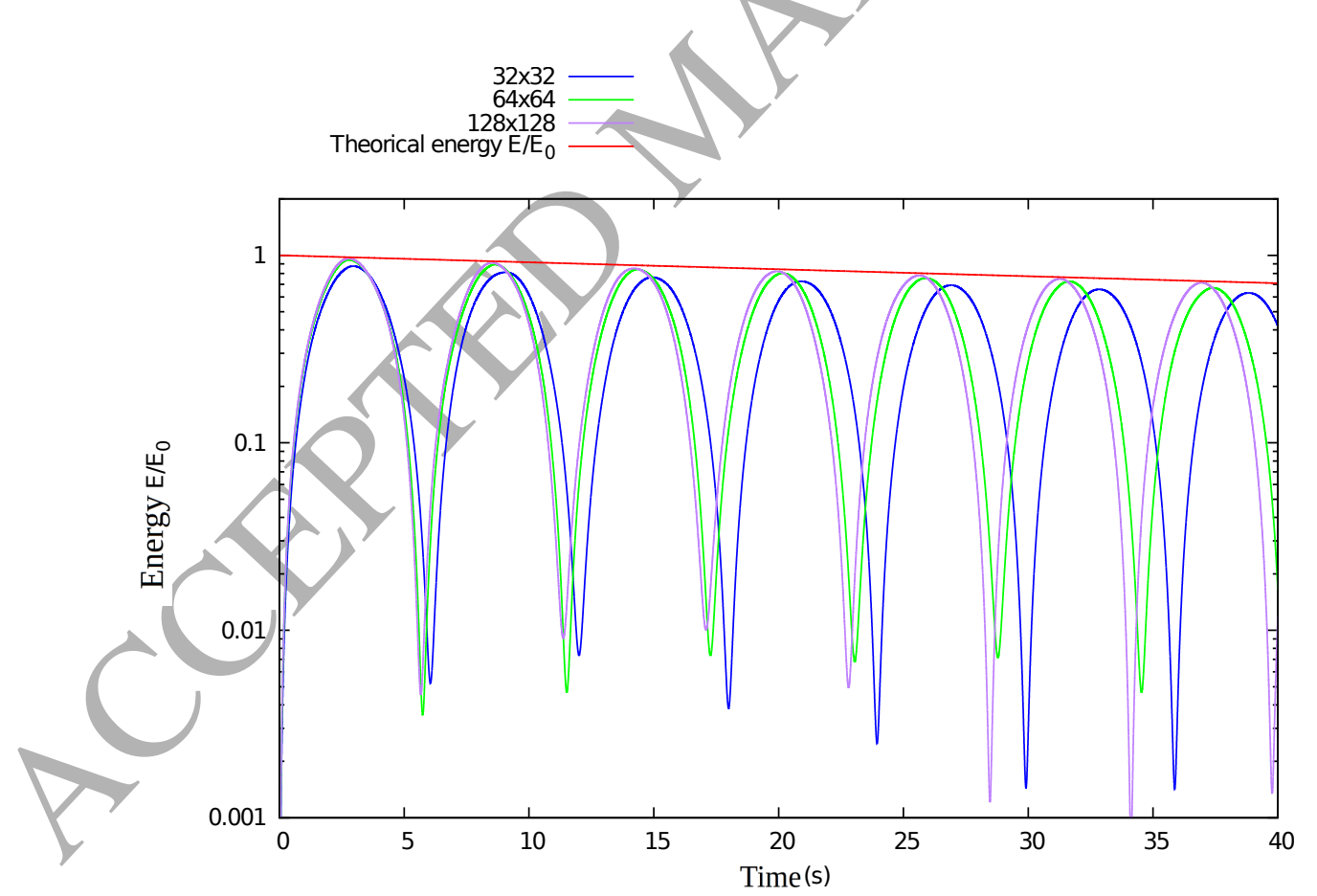

Figure 12: Non-dimensional theorical energy and non-dimensional kinetic energic for the conservating momentum method on single grid

It is interesting to note that the $64^{2}$ grid is almost converged in this case. A brief convergence study is presented graphically on Fig. 12 using the conservative momentum scheme. On $32^{2}$ grid, the energy is 
dissipated too quickly. Closest to the analytical curve, the results on the $64^{2}$ grid shows that the energy decay is slightly overrated. The energy curve of the $128^{2}$ grid is closest to the theoretical curve, showing the capability of the scheme to conserve energy on fine grid. The mesh convergence is successful for energy decay.

We note a shift between curves indicating an error in oscillating period, depending on the mesh size. This error can be estimated by comparison with theoretical value. From the work of Lamb [14], the pulsation of oscillating 2D droplet are (for n-th mode):

$$
w_{n}^{2}=\frac{n(n-1)(n+1)}{\rho_{l}+\rho_{g}} \frac{\sigma}{R^{3}}
$$

The period is calculated by using a relation $T=\frac{2 \pi}{w_{n}}$, we obtain in this case $T=10.99 s$. We understand the oscillation period as a full cycle of horizontal and vertical stretches(every third minimum of kinetic energy).

The Table 3 is then constructed using this theoretical period and data from Fig. 12. From the relative error in Table 3, we conclude that oscillating periods converge correctly. The order of the method is calculated and included in 3 .

\begin{tabular}{|c|c|c|c|}
\hline Mesh & Periods & Relative error & Scheme order \\
\hline $32 \times 32$ & $11.94 \mathrm{~s}$ & 0.0725 & \\
\hline $64 \times 64$ & $11.50 \mathrm{~s}$ & 0.0467 & 0.63 \\
\hline $128 \times 128$ & $11.36 \mathrm{~s}$ & 0.0367 & 0.32 \\
\hline
\end{tabular}

Table 3: Periods of oscillating droplet, compared to (30)

The Table 4 is constructed using energy peaks from Fig. 12 and compare to the theoritical value of energy peak. The order of the method is given in 4 .

\begin{tabular}{|c|c|c|}
\hline Mesh & Relative error & Scheme order \\
\hline $32 \times 32$ & 0.1136 & \\
\hline $64 \times 64$ & 0.0526 & 1.11 \\
\hline $128 \times 128$ & 0.0245 & 1.29 \\
\hline
\end{tabular}

Table 4: Error on Energy peaks

\section{Comparison with experimental data}

Comparison of numerical simulations with experimental data is a big challenge specifically in case of sheared flow with high density ratio. Recently, the development of specific methods (Momentum conservation methods, Velocity extension) allows numerical simulation of realistic configurations. We present two simulations of assisted/atomisation: a 2D sheared liquid sheet and a 3D coaxial injection. Experimental data on these configurations are provided by the LEGI laboratory in Grenoble and they are used to validate our simulations. Note that Fuster et al. [10], Kim et al. [16] et Desjardins et al. [6] have all simulated similar configurations.

\subsection{The sheared liquid sheet}

The simulation is performed in $2 \mathrm{D}$ in order to achieve relatively high resolution. The simulation domain (Fig. 13) is a rectangle of size $L x=8 \cdot 10^{-2} \mathrm{~m}$ and $L y=4 \cdot 10^{-2} \mathrm{~m}$. The mesh is $1024 \mathrm{x} 512$ for a cell size of $78 \mu \mathrm{m}$.

Gas and liquid are injected at $U_{g}=30 \mathrm{~m} / \mathrm{s}$ and $U_{l}=2.6 \cdot 10^{-1} \mathrm{~m} / \mathrm{s}$ respecetively with initial layer thickness of $H_{g}=10^{-2} \mathrm{~m}$ and $H_{l}=10^{-2} \mathrm{~m}$. At the injector nozzle, flows are separated by a thin plate of thickness $e=1.5 \cdot 10^{-4} \mathrm{~m}$ beveled by a $\alpha=3.5^{\circ}$ angle. Fluids properties of water and air are given in the Table 5 . 


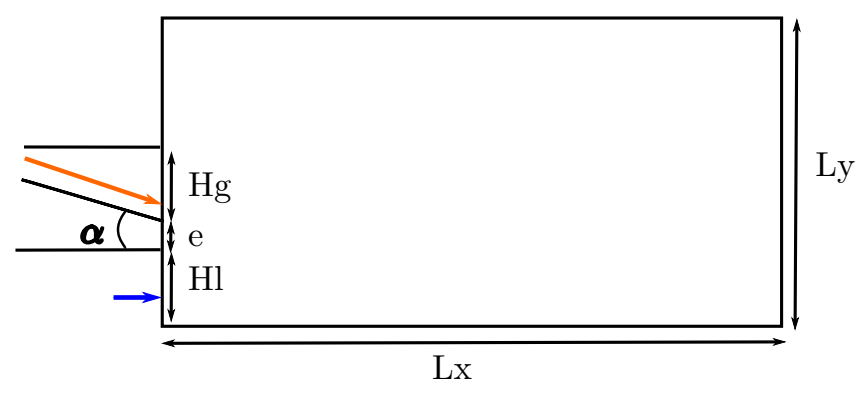

Figure 13: Experimental configuration

\begin{tabular}{|c|c|c|}
\hline & water & air \\
\hline$\rho\left(\mathrm{kg} \cdot \mathrm{m}^{-3}\right)$ & 1000 & 1.2 \\
\hline$\mu\left(\mathrm{kg} \cdot \mathrm{m}^{-1} . \mathrm{s}^{-1}\right)$ & $1.002 \cdot 10^{-3}$ & $1.8 \cdot 10^{-5}$ \\
\hline$\sigma\left(\mathrm{N} \cdot \mathrm{m}^{-1}\right)$ & \multicolumn{2}{|c|}{$7.2 \cdot 10^{-3}$} \\
\hline
\end{tabular}

Table 5: Physical properties of water and air

As previously observed in jet atomization simulations (Cousin et al. [3]), boundary layers have a strong influence on the instabilities development. To be consistent with the experiment [29] at the channel entrance, the velocity field is defined as follow in order to fit with experimental profiles given by [29].

$$
\begin{aligned}
& u= \begin{cases}0.26 \mathrm{~m} / \mathrm{s} & \\
0 \mathrm{~m} / \mathrm{s} & \text { if } y<H l \\
\text { if } H l<y<H l+e \\
\left.U_{g} \cos \left(\frac{\alpha}{2}\right) \operatorname{erf}\left(\frac{y-H l}{0.0007826}\right)\right) & \text { if } H l+e<y<H l+H g+e \\
0 & \text { if } H l+H g+e<y\end{cases} \\
& v= \begin{cases}0 \mathrm{~m} / \mathrm{s} \\
0 \mathrm{~m} / \mathrm{s} \\
U_{g} \sin \left(\frac{\alpha}{2}\right) \operatorname{erf}\left(\frac{y-H l}{0.0007826}\right) & \text { if } y<H l \\
0 & \text { if } H l<y<H l+e \\
\text { if } H l+e<y<H l+H g+e \\
\text { if } H l+H g+e<y\end{cases}
\end{aligned}
$$

where $\operatorname{erf}()$ is the error function.

Simulations are carried out on 512 cores, and we reach 0.3 s physical time with $2.7 \cdot 10^{6}$ time steps. While that configuration led previously to breakdown of the code, the new method does not show any numerical instabilities. 


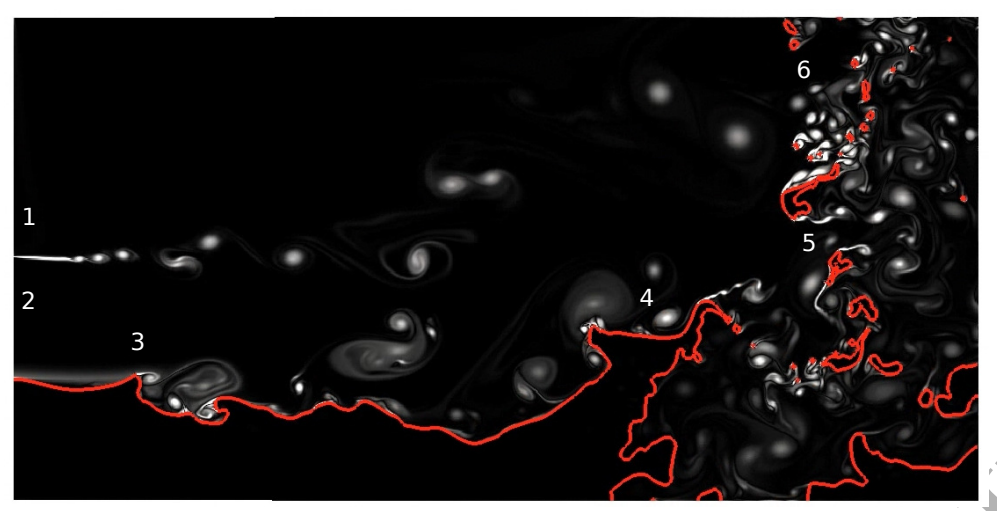

Figure 14: Magnitude of vorticity and interface representation

Qualitative observation is presented in Fig. 14, the vorticity magnitude is represented in greyscale and the interface is the red line. Note that a x-velocity jump between area $1(0 \mathrm{~m} / \mathrm{s})$ and area $2(30 \mathrm{~m} / \mathrm{s})$ gives rise to strong vorticity which generates structures by means of a Kelvin-Helmholtz type instability. As it is observed in experiments $([29],[5],[24])$, some instabilities appear close to the outlet of the injector $\left(\mathrm{n}^{0} .3\right.$ on Fig. 14 ). Further on, they intensify leading to wave formation $\left(n^{0}\right.$. 4$)$. At the top of the waves, some ligaments detach with a large ejection angle $\left(\mathrm{n}^{0} .5\right)$. These are finally broken into small droplets $\left(\mathrm{n}^{0} \cdot 6\right)$. In the wakes of the droplets, a strong vorticity is observed, showing the large inertia of liquid structures.

From analysis of experimental data, Ben Rayana [29] brings to light the presence of a liquid cone which the length can be modeled by :

$M$ is the momentum flux ratio $M=\frac{\rho_{g} U_{g}^{2}}{\rho_{l} U_{l}^{2}}$.
In this configuration $M=16$ and $L_{\text {cone }}=3 \mathrm{~cm}$

$$
L_{\text {cone }}=\frac{12 H_{L}}{\sqrt{M}}
$$

To compare with the modeling approach, we superpose the interface position during 0.2s (Fig. 15). We observe a good agreement with the model. Moreover the graph can be compared to the results of Fuster and al. [11], [20] and Desjardins and al. [6]. The same behaviour is observed in both results. 


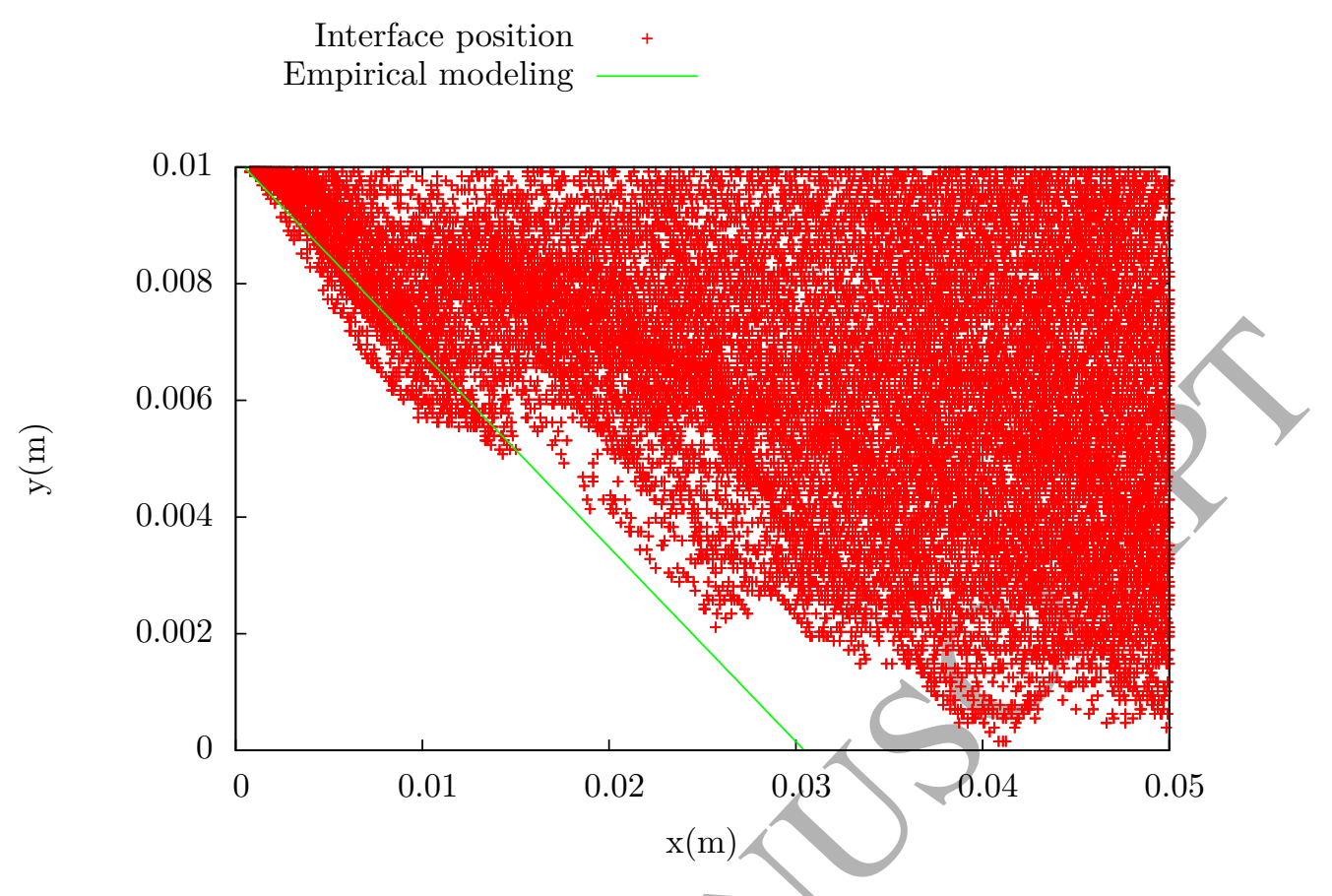

Figure 15: Length of the liquid core from position of interface along time

\subsection{Air assisted atomization: coaxial injector}

In a coaxial-assisted atomization, the liquid is injected at lower velocity than the gas around it. Gas transfers energy to the liquid accelerating it and destabilizing the interface. Many experiments were conducted on these injectors by Rehab and al. [30], Lasheras and al. [17], Marmottant and Villermaux [23] following many parameters at various regimes and for different fluids. They have found eg that when the velocity difference is small, weak disturbances of the interface are observed with small flappering of the liquid, rims propagating and possibly "bags" appearance. Once the velocity difference increases, stripping of the liquid is generated and the atomization process takes place leading to the formation of ligaments and droplets.

The geometric configuration of the numerical simulation is identical to the expriment in LEGI and presented on Fig. 16. The inside diameter measure $D 1=7.6 \cdot 10^{-3} \mathrm{~m}$ and the outside diameter $D 2=$ $1.14 \cdot 10^{-2} \mathrm{~m}$. A lip $e$ of $2 \cdot 10^{-4} \mathrm{~m}$ separates the two flows. The dimensions of the 3D domain are $6.84 \cdot 10^{-2} \mathrm{~m}$ $\mathrm{x} 3.42 \cdot 10^{-2} \mathrm{~m} \times 3.42 \cdot 10^{-2} \mathrm{~m}$. The simulations are performed on a cartesian mesh $1024 \mathrm{x} 512 \mathrm{x} 512$ with a cell size equal to $6.68 \cdot 10^{-5} \mathrm{~m}$. (Higher resolution is time consuming, so only one configuration is considered.) The parameters are chosen with respect to experimental data. Fluids properties of water and air are given in Table 5. The injection velocity of the liquid is $0.26 \mathrm{~m} / \mathrm{s}$ and a flat profile is assumed at the inlet. Gas velocities are taken from experimental measurements at the nozzle exit (Delon [4]). The following inlet profile are defined, with respect to the experimental boundary layer and mean velocity data:

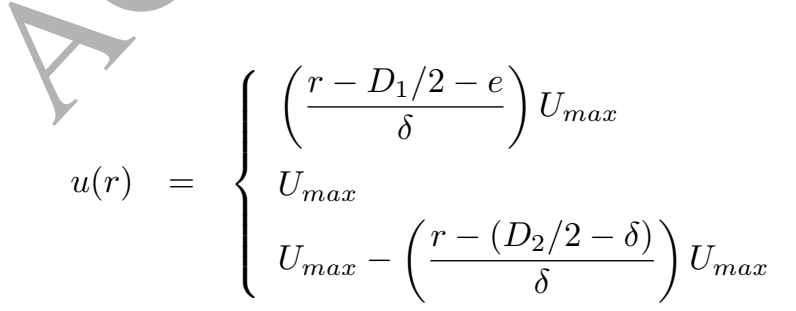

$$
\begin{aligned}
& \text { if } r>\frac{D_{1}}{2}+e \quad \text { and } \quad r<\frac{D_{1}}{2}+e+\delta \\
& \text { if } r>\frac{D_{1}}{2}+e+\delta \quad \text { and } \quad r<\frac{D_{2}}{2}-\delta \\
& \text { if } r>\frac{D_{2}}{2}-\delta \quad \text { and } \quad r<\frac{D_{2}}{2}
\end{aligned}
$$




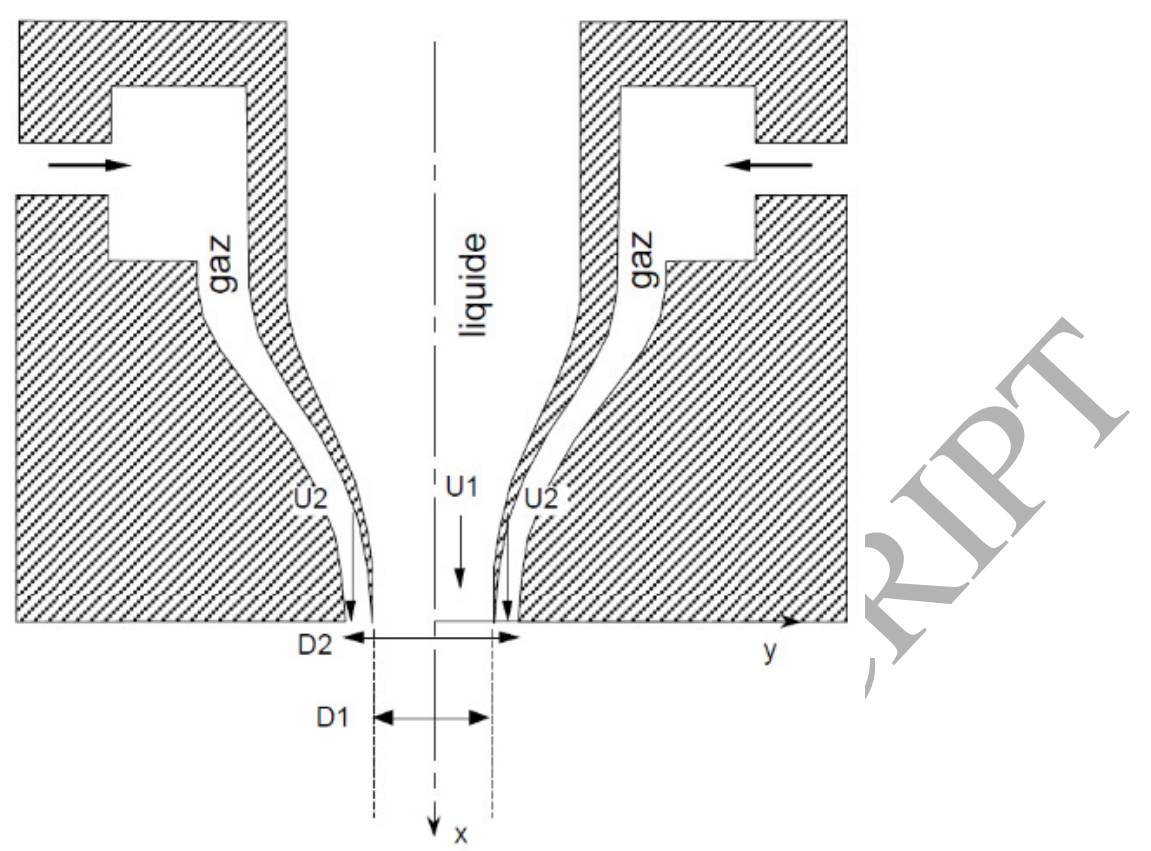

Figure 16: Diagram of the injector used for the experiments at LEGI

where $\delta=2 \cdot 10^{-4} \mathrm{~m}$ is the boundary layer thickness.

$U_{\max }=25 \mathrm{~m} / \mathrm{s}$ is the maximum gas velocity.

As previously observed with the $2 \mathrm{D}$ sheared sheet, in this $3 \mathrm{D}$ coaxial configuration at similarly high density ratio, the solver is stable, and non-physical velocity magnitude never appeared. In Fig. 19, experimental and numerical pictures have been combined, showing macroscopic consistency of numerical results.

Angle of the spray at the outlet of the injector has been evaluated [4] (Fig. 17 a). To obtain it, the authors performed a superposition of pictures along time. The procedure is simple, a pixel is colored black if in at least one of the images the liquid has been recorded there. We apply the same method to our numerical data (Fig. $17 \mathrm{~b}$ ). The angle is relatively well simulated but, nevertheless, we observe it further from the injector outlet.

Employing a similar procedure, the length of the liquid core was evaluated [4] (Fig. 18 a). In this case, a pixel is colored black only if on all the image the liquid has been recorded there. The procedure was reproduced on our numerical pictures (Fig. $18 \mathrm{~b}$ ). The length of liquid core measured in our numerical results is well captured. Extended comparisons between experiments and simulations will be published soon. 


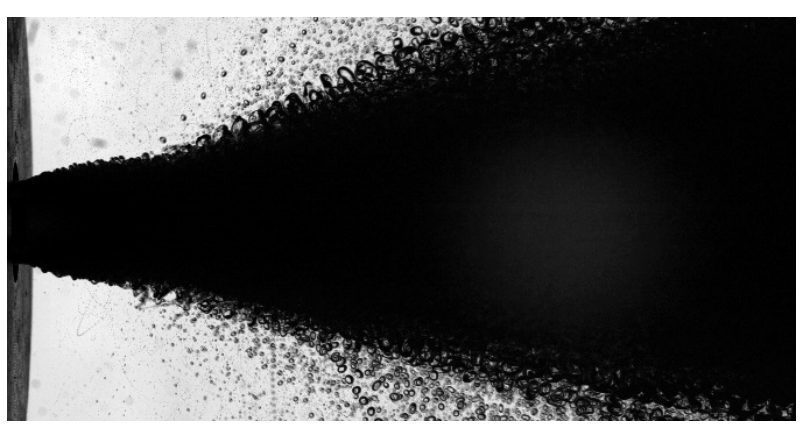

$a$ - Experimental data to estimate ejection angle

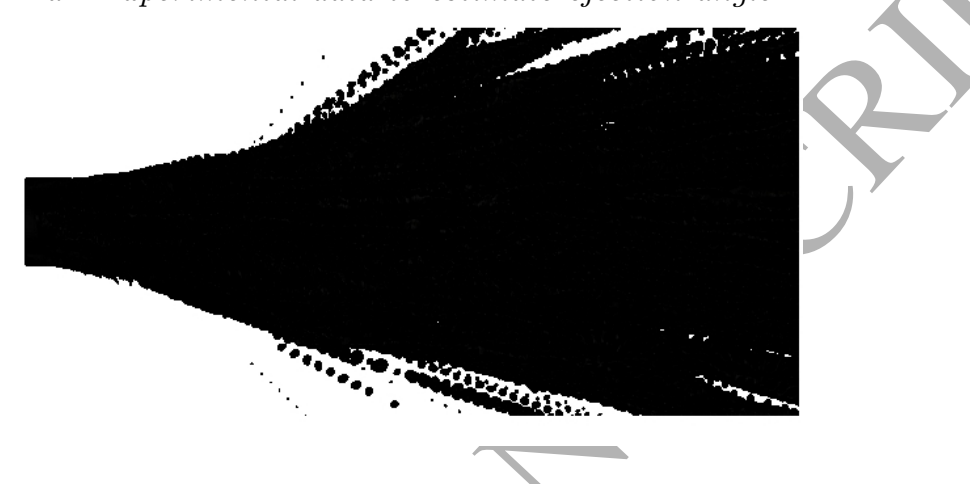

$b$ - Numerical results to estimate ejection angle

Figure 17: Comparison between numerical simulation and experimental results on the ejection angle

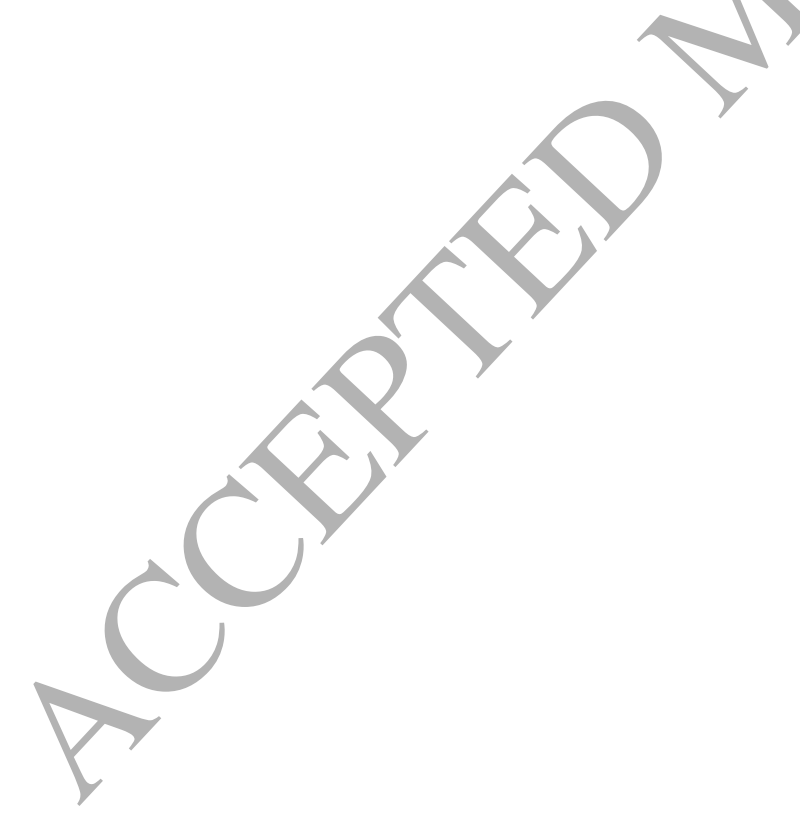




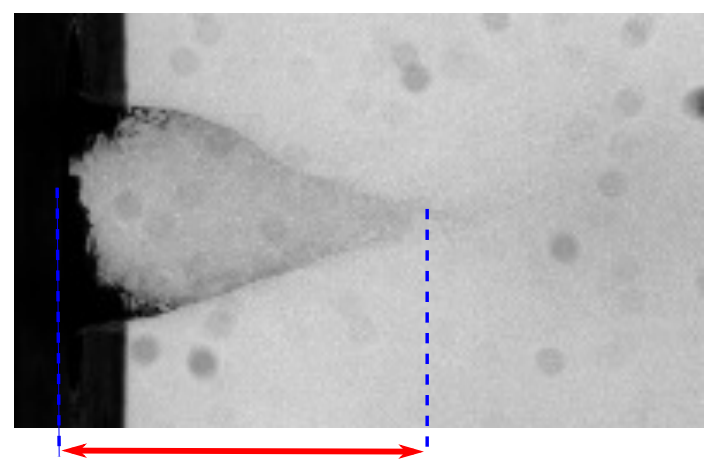

$12.1 \mathrm{~mm}$

a - Length of liquid core from experimental data.

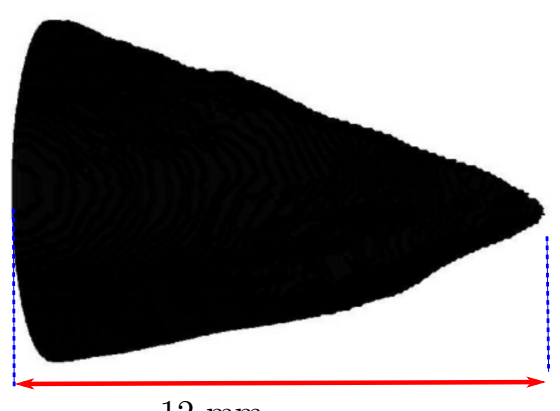

$12 \mathrm{~mm}$

$b$ - Length of liquid core in numerical simulation.

Figure 18: Length of liquid core.

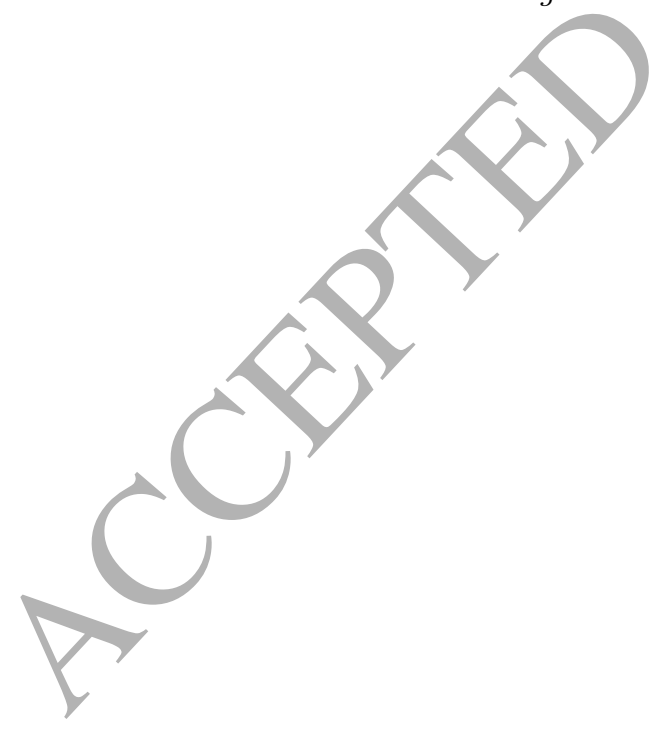




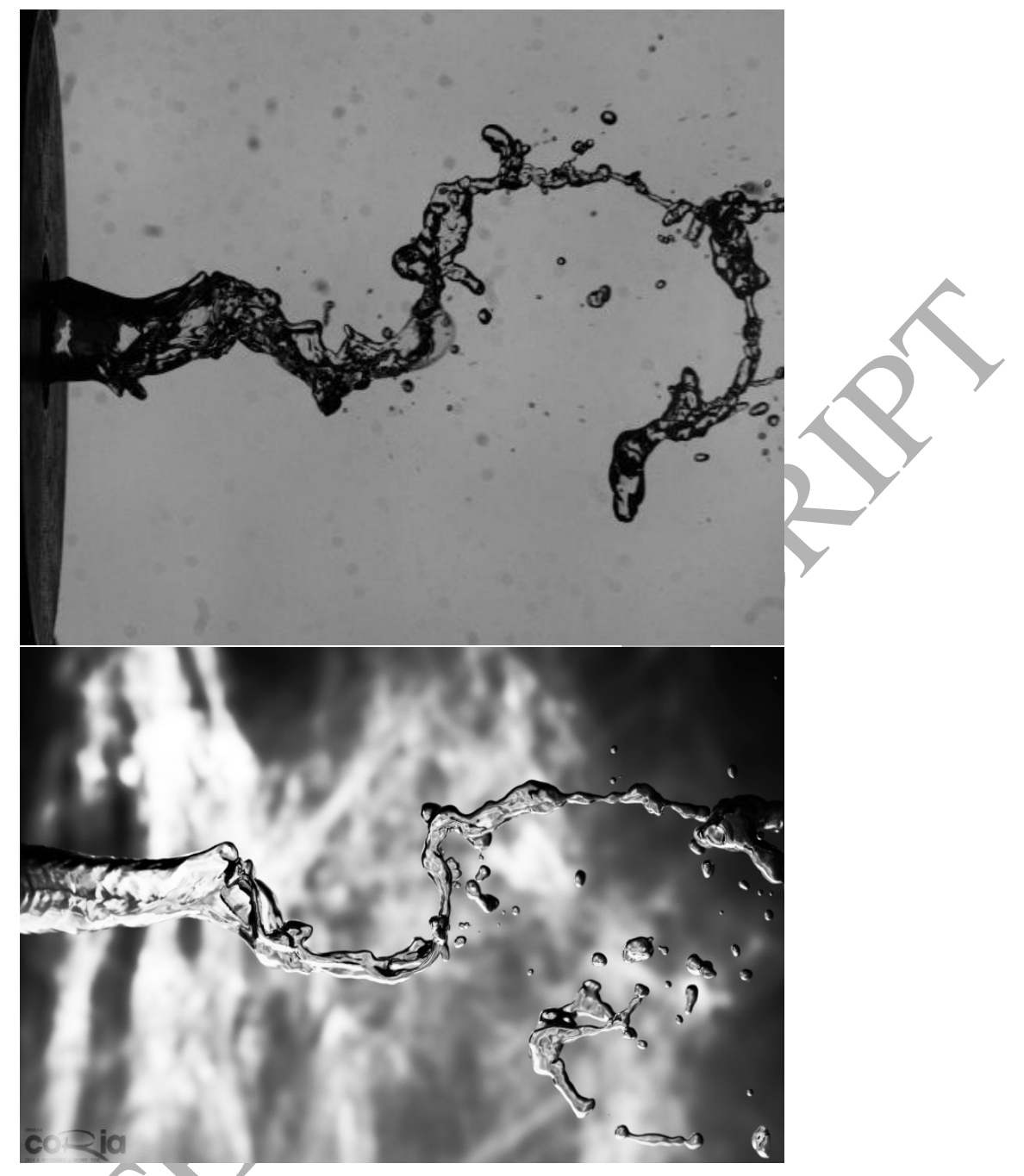

Figure 19: Comparison between numerical simulation and experimental results on the interface topology.

\section{Conclusions}

When modelling two phase flows, high density ratio coupled to high sheared flows - characteristic e.g. of assisted atomization - is a challenge. It has been shown, that the widespread MAC approach introduces significant numerical errors, leading in turn to unstability of the solver or non-physical velocity values. The source of the problem is modelling of convective term in a nonconservative fashion, which causes unexpected energy transfers between modelled phases.

Following the idea of Rudman, the conservative momentum method has been adapted to CLSVOF interface tracking - without subgrid - to be compatible with our Archer solver. To obtain the consistency between mass and momentum transport, a new method to evaluate all mass fluxes has been presented. Once known, the fluxes are then introduced into the convective term calculation. As shown in a number of test cases, the newly presented conservative-momentum method has greatly improved the momentum flux calculation, thus ensuring consistency between mass and momentum transport. This allowed us to simulate two example configurations of assisted liquid atomization: with realistic density ratios which were unfeasible before. The outcome is not only stable behaviour of our solver: also the visualized results, by means of 
qualitative comparison with experimental results, have proven realistic. Also each of the quantitative we have measured shows a good agreement with the experimental data.

\section{Acknowledgments}

Part of this work has been supported by National Research Agency (ANR) program Investissements d'Avenir : ANR VAA (Vistual Assisted Atomisation), ANR MODEMI (MODElisation et simulation Multiéchelle des Interfaces), ANR-10-LABX-09-01 EMC3-TUVECO (Turbulence et viscoélasticité dans les Ecoulements COmplexes)

Computations have been carried out in IDRIS-CNRS (Institut du Développement et des Ressources en Informatique Scientifique), Curie TGCC (Très Grand Centre de Calcul CEA) and CRIANN (Centre Régional Informatique et d'Applicatios Numériques de Normandie)

\section{References}

[1] W. Aniszewski, T. Ménard, and M. Marek. Volume of fluid (vof) type advection methods in two-phase flow: A comparative study. Computers \& Fluids, 97(0):52 - 73, 2014.

[2] T.D. Aslam. A partial differential equation approach to multidimensional extrapolation. Journal of Computational Physics, 193(1):349 - 355, 2004.

[3] Jean Cousin, Alain Berlemont, Thibaut Ménard, and Sébastien Grout. Primary breakup simulation of a liquid jet discharged by a low-pressure compound nozzle. Computers and Fluids, 63(0):165 - 173, 2012.

[4] Antoine Delon, Jean-Philippe Matas, and Alain Cartellier. Flapping instability of a liquid jet. In 8th International Conference on Multiphase Flow, ICMF 2013, Jeju, Korea, May 26 - 31, 2013, pages ICMF2013-586, South Korea, May 2013.

[5] M.N. Descamps, J.P. Matas, and A. Cartellier. Gas-liquid atomisation: gas phase characteristics by piv measurements and spatial evolution of the spray. In 2nd Colloque INCA, Initiative en Combustion Avancée,, 2008.

[6] O. Desjardins, J. McCaslin, M. Owkes, and P. Brady. Direct numerical and large-eddy simulation of primary atomization in complex geometries. Atomization and Sprays, 23(11):1001-1048, 2013.

[7] O Desjardins and V Moureau. Methods for multiphase flows with high density ratio. Proceedings of the Summer Program, 2010.

[8] Ronald P Fedkiw, Tariq Aslam, Barry Merriman, and Stanley Osher. A non-oscillatory eulerian approach to interfaces in multimaterial flows (the ghost fluid method). Journal of Computational Physics, 152(2):457 - 492, 1999.

[9] Marianne M. François. Recent numerical and algorithmic advances within the volume tracking framework for modeling interfacial flows. Procedia \{IUTAM\}, 15:270 - 277, 2015. \{IUTAM\} Symposium on Multiphase Flows with Phase Change: Challenges and Opportunities.

[10] D. Fuster, G. Agbaglah, C. Josserand, S. Popinet, and S. Zaleski. Numerical simulation of droplets, bubbles and waves: state of the art. Fluid Dynamics Research, 41(6):065001, 2009.

[11] D. Fuster, J.-P. Matas, S. Marty, S. Popinet, J. Hoepffner, A. Cartellier, and S. Zaleski. Instability regimes in the primary breakup region of planar coflowing sheets. Journal of Fluid Mechanics, 736:150-176, 122013.

[12] S Ghods and M Herrmann. A consistent rescaled momentum transport method for simulating large density ratio incompressible multiphase flows using level set methods. Physica Scripta, 2013(T155):014050, 2013.

[13] Denis Gueyffier, Jie Li, Ali Nadim, Ruben Scardovelli, and Stéphane Zaleski. Volume-of-fluid interface tracking with smoothed surface stress methods for three-dimensional flows. Journal of Computational Physics, 152(2):423 - 456, 1999.

[14] Lamb H. Hydrodynamics. Cambridge University Press, 1975.

[15] Guang-Shan Jiang and Chi-Wang Shu. Efficient implementation of weighted \{ENO\} schemes. Journal of Computational Physics, 126(1):202-228, 1996.

[16] D. Kim and P. Moin. Numerical simulation of a round liquid jet using the refined level set grid method with subgrid lagrangian drop breakup model. In American Physical Society, 64th Annual Meeting of the APS Division of Fluid Dynamics, 2011.

[17] J.Q. Lasheras and E.J. Hopfinger. Liquid jet instability and atomization in a coaxial gas stream. Annual Review of Fluid Mechanics, 32(1):275-308, 2000.

[18] Vincent Le Chenadec and Heinz Pitsch. A monotonicity preserving conservative sharp interface flow solver for high density ratio two-phase flows. J. Comput. Phys., 249:185-203, September 2013.

[19] X. Li, M. Arienti, and M. Soteriou. Towards an efficient high-fidelity methodology for liquid jet atomization computations. 48th AIAA Aerospace Sciences Meeting Orlando, pages 105-228, 2010.

[20] Y. Ling, S. Zaleski, and R. Scardovelli. Multiscale simulation of atomization with small droplets represented by a lagrangian point-particle model. International Journal of Multiphase Flow, 76:122 - 143, 2015.

[21] J. Lopez, J. Hernandez, P. Gomez, and F. Faura. An improved plic-vof method for tracking thin fluid structures in incompressible two-phase flows. Journal of Computational Physics, 208(1):51 - 74, 2005. 
[22] Bussmann M, Kothe DB, and Sicilian JM. Modeling high density ratio incompressible interfacial. flows engineering division summer meeting, montreal, canada. Proceedings of ASME 2002, 2002.

[23] P. Marmottant and E. Villermaux. Atomisation primaire dans les jets coaxiaux. Combustion, 2:1-37, 2002.

[24] J.P. Matas, S. Marty, and A. Cartellier. Experimental and analytical study of the shear instability of a gas-liquid mixing layer. Physics of Fluids (1994-present), 23(9):-, 2011.

[25] T. Ménard, S. Tanguy, and A. Berlemont. Coupling level set/vof/ghost fluid methods: Validation and application to 3d simulation of the primary break-up of a liquid jet. International Journal of Multiphase Flow, 33(5):510 - 524, 2007.

[26] M. Raessi and H. Pitsch. Modeling interfacial flows characterized by large density ratios with the level set method. Center for Turbulence Research Annual Research Briefs 2009, 2009.

[27] M. Raessi and H. Pitsch. A level-set based methodology for modeling interfacial flows characterized by large density ratios. ILASS-Americas 22nd Annual Conference on Liquid Atomization and Spray Systems, 2010.

[28] Mehdi Raessi and Heinz Pitsch. Consistent mass and momentum transport for simulating incompressible interfacial flows with large density ratios using the level set method. Computers and Fluids, 63(0):70 - 81, 2012.

[29] F. Ben Rayana. Contribution $\tilde{A}$ l'étude des instabilités interfaciales liquide-gaz en atomisation assistée et taille de gouttes. PhD thesis, 2007.

[30] H. Rehab, E. Villermaux, and E. J. Hopfinger. Flow regimes of large-velocity-ratio coaxial jets. Journal of Fluid Mechanics, 345(1):357-381, 1997.

[31] Murray Rudman. A volume-tracking method for incompressible multifluid flows with large density variations. International Journal for Numerical Methods in Fluids, 28(2):357-378, 1998.

[32] B.M. Rush and A. Nadim. The shape oscillations of a two-dimensional drop including viscous effects. Engineering Analysis with Boundary Elements, 24(1):43 - 51, 2000.

[33] M. Sussman, K.M. Smith, M.Y. Hussaini, M. Ohta, and R. Zhi-Wei. A sharp interface method for incompressible two-phase flows. Journal of Computational Physics, 221(2):469 - 505, 2007.

[34] Mark Sussman and Elbridge Gerry Puckett. A coupled level set and volume-of-fluid method for computing 3d and axisymmetric incompressible two-phase flows. Journal of Computational Physics, 162(2):301 - 337, 2000.

[35] G. Vaudor, A. Berlemont, T. Ménard, and M. Doring. A consistent mass and momentum flux computation method using rudman-type technique with a clsvof solver. In Proceedings of the ASME 2014 4th Joint US-European Fluids Engineering, 2014.

[36] F. Xiao, M. Dianat, and J.J. McGuirk. \{LES\} of turbulent liquid jet primary breakup in turbulent coaxial air flow. International Journal of Multiphase Flow, 60(0):103 - 118, 2014.

[37] Jun Zhang. Acceleration of five-point red-black gauss-seidel in multigrid for poisson equation. Applied Mathematics and Computation, 80(1):73 - 93, 1996. 